\title{
Computational Systems Pharmacology, Molecular Docking and Experiments Reveal the Protective Mechanism of Li-Da-Qian Mixture in the Treatment of Glomerulonephritis
}

\author{
Wei Zhou (1D) ${ }^{1,2, *}$ \\ Yugen $\mathrm{Sha}^{2, *}$ \\ Jingxia Zeng ${ }^{3, *}$ \\ Xiaoyue Zhang ${ }^{4}$ \\ Aihua Zhang ${ }^{1,2}$ \\ Xuhua $\mathrm{Ge}^{1,3}$
}

'Nanjing Key Laboratory of Pediatrics, Children's Hospital of Nanjing Medical University, Nanjing, People's Republic of China; ${ }^{2}$ Department of Nephrology, Children's Hospital of Nanjing Medical University, Nanjing, People's Republic of China; ${ }^{3}$ Pediatric Intensive Care Unit, Children's Hospital of Nanjing Medical University, Nanjing, People's Republic of China; ${ }^{4}$ Department of Chinese Medicine, The First Affiliated Hospital of Anhui University of Chinese Medicine, Hefei, People's Republic of China

*These authors contributed equally to this work
Correspondence: Xuhua Ge

Pediatric Intensive Care Unit, Children's Hospital of Nanjing Medical University, 72 Guangzhou Road, Nanjing, 210029.

People's Republic of China

Email gexuhua@njmu.edu.cn

Aihua Zhang

Department of Nephrology, Children's Hospital of Nanjing Medical University, 72 Guangzhou Road, Nanjing, 210029,

People's Republic of China

Email zhaihua@njmu.edu.cn
Background: Glomerulonephritis is a common urinary system disease among children. Growing evidence suggests that traditional Chinese medicine has potential in treating glomerulonephritis, such as Li-Da-Qian mixture. Although its anti-glomerulonephritis and alleviating hematuria effects have been reported, the exact mechanism of Li-Da-Qian mixture devoting to glomerulonephritis remains unexplored. It was necessary to explore the mechanism of Li-Da-Qian mixture against glomerulonephritis using modern technology, such as Chinese medicine database and molecular biological experiments.

Methods: Online databases were used to look up ingredients and predict targets of Li-Da-Qian mixture against glomerulonephritis. The intersecting targets of Li-Da-Qian mixture and glomerulonephritis were selected for enrichment analysis. Cytoscape software was applied to establish network and MCODE analysis. Molecular docking was used for the primary validation. Furthermore, we examined the function of the core compounds analyzed from Li-Da-Qian mixture to rescue LPS-induced inflammation in vivo and vitro. We also explored whether the core compounds can alleviate TGF $\beta 1$-induced renal fibrosis in mouse proximal tubular cells.

Results: Network pharmacological analysis of Li-Da-Qian evaluated 20 active ingredients including baicalein, luteolin and quercetin. A total of 113 key targets were screened, including IL6, VEGFA, TP53, EGF, MMP2, etc, and they were enriched in AGE-RAGE signaling pathway in diabetic complications, TNF and IL-17 signaling pathways. Moreover, the core ingredients succeeded in binding to the main targets via molecular docking, further identifying the anti-glomerulonephritis effects and improvement of vascular injury. Western blotting and qPCR also suggested that baicalein and luteolin can improve inflammation and restore disturbance of mesangial cells or kidney induced by LPS. In addition, baicalein and luteolin inhibited renal fibrosis in vitro.

Keywords: glomerulonephritis, network pharmacology, Li-Da-Qian mixture, traditional Chinese medicine, molecular docking

\section{Introduction}

Glomerulonephritis is one of the most common urinary system diseases, which is associated with environmental changes, genetic predisposition and immune disorder. ${ }^{1}$ The accumulation of immune complexes in the kidney leads to the activation of effector immune cells, the release of cytokine and the secretion of enzymes including matrix metalloproteinases (MMPs). ${ }^{2-4}$ The above substances make a concerted effort to damage glomerular capillary tuft. Acute post-streptococcal glomerulonephritis (APSGN) which is 
usually seen in children's hospital mainly occurs in pediatric age group and primarily occurs in developing countries. ${ }^{5,6}$ Pediatric patients suffering from APSGN usually completely recover. However, when the kidney presents abundant crescents, rare APSGN cases can evolve into chronic kidney disease, which is an independent risk factor resulting in end-stage kidney disease. ${ }^{7,8}$ Therefore, pediatricians should pay more attention to APSGN to prevent renal malignant development. Antibodies, renal biopsy and immunosuppression therapy are not routinely warranted in APSGN. ${ }^{7}$ At present, there is no specific treatment for APSGN, so new powerful and effective drugs need to be developed.

Traditional Chinese medicine makes great contributions to treating kidney diseases, which is famous for mild nature and less side effects. For instance, Huang Qi Huai $(\mathrm{HQH})$ granules, which contain polygonatum, trametes robiniophila murr and wolfberry fruit, can attenuate nephrotic syndrome and postpone the development of human primary glomerular diseases. ${ }^{9,10}$ Celastrol extracted from Chinese medicine Tripterygium wilfordii protects against acute kidney injury derived from cisplatin, probably through stabilizing mitochondrial function and suppressing NF- $\mathrm{B}$ signaling. ${ }^{11}$ Doctors from Nanjing children's hospital use Li-Da-Qian mixture to treat acute glomerulonephritis, which is a kind of Chinese medicine mixture made by our hospital. The constituent herbs in Li-Da-Qian mixture are comprised of $500 \mathrm{~g}$ LIZHICAO (common sage), 250g DAJI (all-grass of Thistle), 250g XIAOJI (Setose Thistle) and 500g CHEQIANCAO (all-grass of rippleseed plantain) which were extracted, concentrated, and dissolved in $1000 \mathrm{~mL}$ purified water. The mixture has been used to attenuate hematuria resulting from APSGN for over fifty years. A study from Ma found that Li-Da-Qian mixture could reduce edema, recover blood pressure and accelerate the time to start diuresis in APSGN patients. ${ }^{12}$ To clarify the mechanism of Li-Da-Qian mixture, modern technology is needed to provide a stable scientific basis, such as computational systems pharmacology, Western blotting and qPCR. ${ }^{13}$ The study is aimed to prove the healing effects and underlying mechanism of Li-Da-Qian mixture.

\section{Materials and Methods}

The Collection of Active Ingredients in LiDa-Qian Mixture

To find as many active ingredients in Li-Da-Qian mixture as possible, we searched Traditional Chinese Medicine Systems Pharmacology Database and Analysis Platform
(TCMSP, https://tcmsp-e.com/), Traditional Chinese Medicine Database@Taiwan (TCM@Taiwan, http://tcm. cmu.edu.tw/), BATMAN-TCM (http://bionet.ncpsb.org. cn/batman-tcm/), Traditional Chinese Medicines Integrated Database (TCMID, http://www.megabionet. org/tcmid/) and The Encyclopedia of Traditional Chinese Medicine (http://www.tcmip.cn/ETCM/index.php/Home/ Index/index.html), with the keywords Lizhicao, Daji, Xiaoji and Cheqiancao respectively. TCMSP is a professional systems pharmacology platform of Chinese herbal medicines consisting of 449 herbs for looking up the inner relationship among drugs, targets and diseases. ${ }^{14}$ TCMSP has two key indexes called oral bioavailability (OB) and drug-like (DL), which is the reason for being applied preferentially. OB is the fraction of a dose that reaches the blood circulation for oral drugs reflecting pharmacokinetic characteristics. ${ }^{15}$ DL is used to judge whether investigative ingredients are similar with reported compounds. ${ }^{15}$ It is generally acknowledged that the ingredients meeting $\mathrm{OB}>30 \%$ and $\mathrm{DL}>0.18$ are recognized active compounds and suitable for the next analysis. ${ }^{14}$ TCM@Taiwan, BATMAN-TCM, TCMID and ETCM database do not include $\mathrm{OB}$ and DL, but they can be used for searching some other ingredients and targets that are not found in TCMSP.

\section{The Retrieval of Relative Targets of Active Ingredients of Li-Da-Qian Mixture and Glomerulonephritis}

TCMSP database was taken for prior for retrieving potential targets of active ingredients. Additionally, UniProt (https://www.uniprot.org) is used to annotate gene names from TCMSP. Related targets of glomerulonephritis are obtained from Online Mendelian Inheritance in Man (OMIM, https://www.omim.org/) and Genecards (https:// www.genecards.org/). ${ }^{16,17}$ OMIM and Genecards are websites integrating the information between diseases and genes, which were used for finding reported targets of glomerulonephritis.

\section{The Electronic Drawing of Ingredient- Target-Disease Network}

Both active ingredients and corresponding targets were input into Cytoscape 3.6.1 (https://cytoscape.org/) to establish ingredient-target-disease network. The core ingredients were obtained from the above network. 
The Use of Target Genes of Li-Da-Qian Mixture to Treat Glomerulonephritis for Analyzing Protein-Protein Interaction (PPI) Network, Gene Ontology (GO) and Kyoto Encyclopedia of Genes and Genomes (KEGG)

STRING (https://string-db.org/) is a free public database widely used to construct the network of protein-protein interaction (PPI) ${ }^{18}$ STRING database can calculate each interaction scores between targets. The scores are directly proportional to the confidence. The following actions were operated in sequence, choosing "multiple proteins", listing the intersecting genes of Li-Da-Qian mixture and glomerulonephritis, selecting "Homo sapiens" for searching and medium confidence (0.4) was set. GO and KEGG results were achieved as well as PPI network because of built-in function of Integrated Discovery website (DAVID, https:// david.ncifcrf.gov/) in STRING. ${ }^{19}$

\section{Verification of Ingredient-Target Interaction via Molecular Docking}

"Key-Lock" principle is the basic theory of molecular docking, which reflects the association between small-molecule ligands and protein receptors. In this research, the crystal structure files of all proteins were downloaded from the RCSB PDB database (http://www.rcsb.org/). The receptor structure was edited by AutoDockTools with removal of water molecules, adding polar hydrogen atoms, adding Kollman Charges and save PDB file as PDBQT file. The ligand structure was downloaded from PubChem (https:// pubchem.ncbi.nlm.nih.gov/) and it was necessary to perform energy minimization parameters by PyRx. The final docking operation was also edited in PyRx, and the binding energy between ligands and receptors was acquired. When the binding energy of the ingredient-target complex is less than or equal to $-5 \mathrm{KJ} / \mathrm{mol}$, it indicates that the two can spontaneously bind. Finally, both two-dimensional and three-dimensional interactions were presented in Discovery Studio 4.5 software for visualization.

\section{Animal Model}

Wild-type 8-week-old C57BL/6J mice were obtained from Zhejiang Vital River Laboratory Animal Technology Co., Ltd. (Zhejiang, China) and were maintained on a 12-h light and 12-h dark cycle in a standard SPF animal room. LPS (L2880, LPS from E. coli 055:B5) bought from Sigma-
Aldrich (St. Louis, MO, United States) induced kidney injury. Mice were divided into six groups: control group $(\mathrm{n}=6), 10 \mathrm{mg} / \mathrm{kg}$ baicalein treatment group $(\mathrm{n}=6), 20 \mathrm{mg} / \mathrm{kg}$ baicalein treatment group $(\mathrm{n}=6), 30 \mathrm{mg} / \mathrm{kg}$ LPS group $(\mathrm{n}=9)$, $30 \mathrm{mg} / \mathrm{kg} \mathrm{LPS}+10 \mathrm{mg} / \mathrm{kg}$ baicalein group $(\mathrm{n}=9)$ and $30 \mathrm{mg} / \mathrm{kg}$ LPS $+20 \mathrm{mg} / \mathrm{kg}$ baicalein group $(\mathrm{n}=9)$. Baicalein (10 or $20 \mathrm{mg} / \mathrm{kg}$ ) were given by intraperitoneal injection $2 \mathrm{~h}$ before and $12 \mathrm{~h}$ after LPS $(30 \mathrm{mg} / \mathrm{kg})$ intraperitoneal injection, respectively, and the blank control group and LPS group were equally injected with an equal volume of vehicle. At $24 \mathrm{~h}$ after LPS injection, the mice were sacrificed, and then kidney tissues and blood were collected and stored at $-80^{\circ} \mathrm{C}$ or fixed in $4 \%$ paraformaldehyde (PFA).

\section{Histopathological Evaluation}

The middle parts of kidney tissues in paraffin were orderly dehydrated with graded alcohol and embedded in paraffin. The kidney tissues in paraffin were cut into $3 \mu \mathrm{m}$ sections for hematoxylin and eosin staining ( $\mathrm{H} \& \mathrm{E}$ staining). Images were captured with an Olympus BX51 microscope (Olympus, Tokyo, Japan).

\section{Cell Culture and Cell Counting Kit-8 (CCK-8) Assay}

The mouse mesangial cell line (MC) was bought from the China Center for Type Culture Collection. Cells were cultured in DMEM supplemented with 10\% PAN fetal bovine serum in a humidified $5 \% \mathrm{CO} 2$ and $37^{\circ} \mathrm{C}$ atmosphere. Mouse proximal tubular cells (mPTCs) were obtained from American Type Culture Collection (ATCC, Manassas, VA) and cultured in DMEM/F-12 medium supplemented with $10 \%$ PAN fetal bovine serum under the same culture conditions as mesangial cells.

Cell viability was tested with CCK-8 assay kit (KGA317; KeyGen Biotech, China). In brief, 500 MCs were seeded per well in a 96-well plate and treated with luteolin $(0-80 \mu \mathrm{M})$ in medium without serum for $24 \mathrm{~h}$ when confluence reached $60-70 \%$. Then, $10 \mu \mathrm{L}$ CCK-8 reagent was added to the each well, and the culture was incubated for $0.5-2 \mathrm{~h}$ according to the actual changes of color. The absorbance value was detected at $450 \mathrm{~nm}$ with a Multiskan FC microplate reader (Thermo Fisher, Shanghai, China).

\section{Drug Treatment}

After MCs or mPTCs met $60 \%-70 \%$ confluence, they were pretreated with baicalein (MCE, Cat. No. HY- 
N0196) at $20 \mu \mathrm{M}$ or luteolin (MCE, Cat. No. HY-N0162) at $5 \mu \mathrm{M}$ for $2 \mathrm{~h}$, and then treated with LPS (Sigma, L2280) at $20 \mu \mathrm{g} / \mathrm{mL}$ or human recombinant TGF $\beta 1$ (240-B, R\&D company) at $10 \mathrm{ng} / \mathrm{mL}$ for $24 \mathrm{~h}$.

\section{Quantitative Real-Time PCR}

TRIzol reagent (TaKaRa) was employed to lyse cells for extracting RNA. Reverse transcription depended on a PrimeScript RT reagent Kit (TaKaRa) according to the normal protocol. Primers were synthesized by Tsingke Biotech (Nanjing, China). Real-time PCR amplification was performed using the LightCycler ${ }^{\circledR} 96$ Real-time PCR System by using SYBR Premix Ex Taq (TaKaRa). The following primer sequences were used: Mouse TNF- $\alpha, 5^{\prime}-$ TCCCCAAAGGGATGAGAAG-3' and reverse, 5'-CAC TTGGTGGTTTGCTACGA-3'; Mouse IL-1 $\beta, 5^{\prime}$-ACTGT GAAATGCCACCTTTTG-3' and reverse, 5'-TGTTGA TGTGCTGCTGTGAG-3'; Mouse iNOS, 5'-ACTCAGCC AAGCCCTCACCTAC-3' and reverse, 5'-TCCAATCTCT GCCTATCCGTCTCG-3'; Mouse IL-6, 5'-ACAAAGCC AGAGTCCTTCAGAGAG-3' and reverse, 5'-TTGGATG GTCTTGGTCCTTAGCCA-3'; Mouse MMP2, 5'-CAA GTTCCCCGGCGATGTC-3' and reverse, 5'-TTCTGGT CAAGGTCACCTGTC-3'; Mouse MMP9, 5'-CATTCGC GTGGATAAGGAGT-3' and reverse, 5'-ACCTGGTT CACCTCATGGTC-3'; Mouse IL10, 5'-GCTCTTACTG ACTGGCATGAG-3' and reverse, 5'-CGCAGCTCTAGG AGCATGTG-3'. Mouse FN, 5'-CGTGGAGCAAGAAG GACAA-3' and reverse, 5'-GTGAGTCTGCGGTTGGT AAA-3'; Mouse $\alpha$-SMA, 5'-CCCTGAAGAGCATCCG ACA-3' and reverse, 5'-CCAGAGTCCAGCACAATA CC-3'; Mouse Collagen I, 5'-CCGGCTCCTGCTCCTCT T-3' and reverse, 5'-TTGCACGTCATCGCACAC - $3^{\prime}$.

\section{Western Blotting Analysis}

The MC cells and mPTCs were lysed in protein lysis buffer $\left(1 \mathrm{mM} \mathrm{Na} \mathrm{VO}_{4}, 2 \mathrm{mM}\right.$ EDTA, $50 \mathrm{mM}$ Tris- $\mathrm{HCl}$, $50 \mathrm{mM} \mathrm{NaF}, 250 \mathrm{mM} \mathrm{NaCl}$ and $0.5 \%$ Triton X-100) and supplemented with $1 \times$ phosphatase inhibitor and $1 \times$ protease inhibitor for half an hour on ice. Western blotting was performed with $40 \mu \mathrm{g}$ total protein and transferred onto PVDF membranes (Bio-Rad). The membranes were blocked for 1 hour at room temperature in TBST containing 5\% (weight/volume) non-fat milk with primary antibodies against iNOS (Abcam, ab3523, 1:1000), NLRP3 (bioss, bs-6655R, 1:1000), NRF2 (proteintech, 16396-1AP, 1:500) and COX-2 (Cayman, aa584-598, 1:500), p-P53 (CST, 9284, 1:1000), P53 (proteintech, 10442-1-
AP, 1:1000), FN (Abcam, ab2413, 1:1000), p-Smad2S465/467+p-Smad3-S423/425 (abclonal, AP0548, 1:1000), Smad2 (abclonal, A19114, 1:1000), Smad3 (abclonal, A19115, 1:1000) by overnight incubation at $4^{\circ} \mathrm{C}$, followed by the addition of HRP-labeled secondary antibodies at room temperature for $2 \mathrm{~h}$. GAPDH/ACTB (proteintech, 1:5000) was used as an internal standard control. Band intensity was measured using ImageJ software (NIH, Bethesda, MD, USA).

\section{Data Analysis}

Data are presented as mean \pm SEM. Two-way analysis of variance (ANOVA) analysis was followed. $P<0.05$ was considered statistically significant. Some figures were conducted by RStudio (1.3.1093), Venny2.1.0 (https://bioin fogp.cnb.csic.es/tools/venny/index.html) and hiplot online website (https://hiplot.com.cn/).

\section{Results \\ Active Compounds of Li-Da-Qian Mixture}

In total, 136 bioactive ingredients were discovered from TCMSP and other databases containing 42 types in Daji, 13 types in Xiaoji, 70 types in Cheqiancao, and 11 types in Lizhicao. The details were shown in the Supplementary file, Figure S1. After selecting OB $>30 \%$, DL $>0.18$ and removing duplicates, 20 active ingredients were totally screened. It included 10 types (MOL001735, MOL001749, MOL002032, MOL002879, MOL003344, MOL000359, MOL000449, MOL005842, MOL005846, MOL000596) in Daji, 5 types (MOL000098, MOL000359, MOL000449, MOL001689, MOL001790) in Xiaoji, 10 types (MOL001735, MOL002714, MOL002776, MOL000359, MOL004004, MOL000449, MOL000006, MOL007783, MOL007796, MOL007799) in Cheqiancao and only 1 type in Lizhicao (Figure 1). The details were shown in the Supplementary file, Table S1.

\section{Potential Target Genes of Li-Da-Qian Mixture and Glomerulonephritis}

In all, 204 target genes of Li-Da-Qian mixture were obtained from TCMSP database. However, six ingredients consisting of beta-amyrin acetate, melampyroside, pectolinarin, stigmasteryl palmitate, taraxasterol acetate and $\beta$ sitosteryl palmitate are unable to find their targets from TCMSP. We used online tool SwissDock to predict these drug targets. ${ }^{20}$ We got the sdf format file of these 


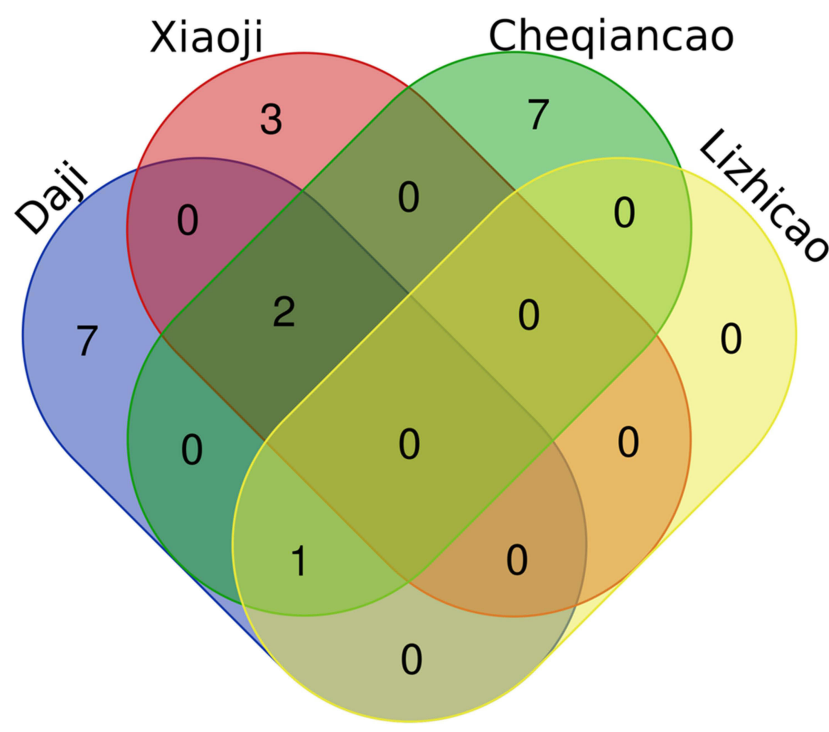

Figure I Twenty core compounds of four herbs from Li-Da-Qian mixture.

compound structures from PubChem. Then, we imported these sdf files into SwissDock (http://www.swissdock.ch/) with selecting "Homo sapiens" for searching predicted targets, among which probability $>0.1$ are chosen. Totally, 244 drug targets were found (Table S2). Moreover, 1782 target genes of glomerulonephritis were totally attained from OMIM and Genecards database. The details were shown in the Supplementary file, Figure S2 and Table S3. Finally, 113 putative targets of Li-Da-Qian mixture were got after integrating 244 putative targets of Li-Da-Qian mixture with 1782 glomerulonephritis gene targets. PTGS1, PTGS2, IL1B, IL2, IL4, IL6, IL10, MMP2, MMP9, etc were directly related targets identified between Li-Da-Qian mixture and glomerulonephritis, and details are shown in Table 1.

\section{Enrichment Analysis}

GO enrichment made contributions to analyzing biological process (BP), cellular component (CC), and molecular function (MF) of the gene targets of Li-Da-Qian mixture antiglomerulonephritis. Altogether 113 potential genes were highly enriched in $1796 \mathrm{BP}, 85 \mathrm{CC}$, and $170 \mathrm{MF}$ terms with p-value $<0.05$. As shown in Figure 2, response to oxygencontaining compound $(\mathrm{p}=1.52 \mathrm{E}-48)$, response to organic substance $(\mathrm{p}=9.26 \mathrm{E}-48)$, cellular response to chemical stimulus $(p=3.43 \mathrm{E}-47)$ and response to abiotic stimulus $(p=1.22 \mathrm{E}-$ 41) were tightly related to glomerulonephritis biological processes. With respect to cellular components, more significant enrichment was found in extracellular space $(\mathrm{p}=2.18 \mathrm{E}-20)$, extracellular region $(\mathrm{p}=1.45 \mathrm{E}-15)$ and intracellular organelle lumen $(\mathrm{p}=1.91 \mathrm{E}-08)$. The main glomerulonephritis-related terms in molecular functions consisted of protein binding $(\mathrm{p}=$ 2.06E-21) and signaling receptor binding $(\mathrm{p}=2.64 \mathrm{E}-20)$ and enzyme binding $(p=5.72 \mathrm{E}-18)$.

KEGG analysis concentrated on the anti-glomerulonephritis signaling pathways of Li-Da-Qian mixture. Results demonstrated that "TNF signaling pathway", "AGE-RAGE signaling pathway in diabetic complications", "IL-17 signaling pathway" and "HIF-1 signaling pathway" were the most associated with the pathogenesis of glomerulonephritis (Table 2 and Figure 3). Additionally, "AGE-RAGE signaling pathway in diabetic complications" was found to be the closest pathway with a high enrichment degree in glomerulonephritis and mapped 31 gene targets. Advanced glycation end product (AGE) accumulation in acute inflammatory glomerulonephritis secondary to systemic lupus erythematosus contributes to glomerular injury, possibly via enzymatic oxidation of glomerular matrix proteins. ${ }^{21}$ From the KEGG map of AGE-RAGE signaling pathway in diabetic complications (https://www.genome.jp/ kegg-bin/show pathway?hsa04933), we can conclude that the activation of AGE-RAGE signaling finally results in mesangial matrix expansion, angiogenesis, thrombogenesis, vascular dysfunction, vascular remodeling, cellular proliferation leading to renal hypertrophy and inflammation. The above pathological process is closely related to the occurrence and development of glomerulonephritis, which indicates that Li-Da-Qian mixture may play a part in glomerulonephritis secondary to diabetic nephropathy.

\section{Pharmacological Network Construction Potential Targets of Li-Da-Qian Network Analysis}

The network construction of active compound-potential targets of Li-Da-Qian mixture using 4 ingredients, 20 compounds and 244 targets was based on the "one to multiple", "multiple to one" links between 275 nodes and 460 edges in this complex network (Figure 4). The more nodes and edges acquired, the stronger the node interaction, and versa vice. In the analyzed network, quercetin $($ degree $=151)$, luteolin $($ degree $=58), \beta$-amyrin acetate $($ degree $=46)$, and baicalein $($ degree $=38)$ were the top active ingredients of Li-Da-Qian mixture for the treatment of glomerulonephritis, which were prepared for molecular docking with targets.

\section{Protein-Protein Interaction Network Construction}

Totally, 113 core genes were imported into STRING for building a PPI network containing 113 nodes and 1967 
Table I Potential Targets of Li-Da-Qian Mixture Against Glomerulonephritis

\begin{tabular}{|c|c|c|c|c|c|}
\hline No. & Gene & No. & Gene & No. & Gene \\
\hline 1 & $R X R A$ & $4 I$ & PPARG & 81 & TGFBI \\
\hline 2 & PTGSI & 42 & $\mathrm{KCNH} 2$ & 82 & IL2 \\
\hline 3 & PTGS2 & 43 & FIO & 83 & PLAT \\
\hline 4 & $A D R B 2$ & 44 & MMP3 & 84 & THBD \\
\hline 5 & $A K R I B I$ & 45 & EGFR & 85 & SERPINEI \\
\hline 6 & PLAU & 46 & AKTI & 86 & COLIAI \\
\hline 7 & LTA4H & 47 & VEGFA & 87 & IFNG \\
\hline 8 & CHRM3 & 48 & CCNDI & 88 & PTEN \\
\hline 9 & HTR2A & 49 & FOS & 89 & ILIA \\
\hline 10 & NOS2 & 50 & MMP2 & 90 & MPO \\
\hline 11 & DPP4 & 51 & MMP9 & 91 & NQOI \\
\hline 12 & HSP9OAAI & 52 & MAPKI & 92 & PARPI \\
\hline 13 & PIK3CG & 53 & ILIO & 93 & COL3AI \\
\hline 14 & PRSSI & 54 & $E G F$ & 94 & CXCLII \\
\hline 15 & $F 2$ & 55 & $R B I$ & 95 & CXCL2 \\
\hline 16 & NOS3 & 56 & CD40LG & 96 & INSR \\
\hline 17 & $\mathrm{RHO}$ & 57 & JUN & 97 & PPARA \\
\hline 18 & $A R$ & 58 & IL6 & 98 & PPARD \\
\hline 19 & $A P O B$ & 59 & $C D K N 2 A$ & 99 & $C R P$ \\
\hline 20 & HMGCR & 60 & ELKI & 100 & CXCLIO \\
\hline 21 & MTTP & 61 & NFKBIA & 101 & SPPI \\
\hline 22 & PREP & 62 & $O D C I$ & 102 & $E 2 F I$ \\
\hline 23 & CYPI9AI & 63 & $X D H$ & 103 & IRFI \\
\hline 24 & $\mathrm{NR} 3 \mathrm{Cl}$ & 64 & SODI & 104 & PONI \\
\hline 25 & CYP2CI9 & 65 & MMPI & 105 & CDK4 \\
\hline 26 & EPASI & 66 & STATI & 106 & PCNA \\
\hline 27 & ALOX5 & 67 & HSPA5 & 107 & IL4 \\
\hline 28 & HIFIA & 68 & HMOXI & 108 & FOSLI \\
\hline 29 & PRKCA & 69 & CYP3A4 & 109 & FOSL2 \\
\hline 30 & C5ARI & 70 & CYPIA2 & 110 & MME \\
\hline 31 & MAPK I 4 & 71 & MYC & 111 & SLC5A2 \\
\hline 32 & $C D K 2$ & 72 & F3 & 112 & SLC5AI \\
\hline 33 & RELA & 73 & GJAI & 113 & $T N F$ \\
\hline 34 & $B C L 2$ & 74 & ICAMI & & \\
\hline 35 & CDKNIA & 75 & $I L I B$ & & \\
\hline 36 & $B A X$ & 76 & CCL2 & & \\
\hline 37 & CASP3 & 77 & SELE & & \\
\hline 38 & TP53 & 78 & VCAMI & & \\
\hline 39 & CASP8 & 79 & CXCL8 & & \\
\hline 40 & FASLG & 80 & $P R K C B$ & & \\
\hline
\end{tabular}

edges (Figure 5A). The main gene targets possessed higher degree and were more likely to play a vital role in Li-DaQian mixture anti-glomerulonephritis containing AKT1, IL6, VEGFA, TNF, TP53, JUN, EGF, MAPK1 and MMP9. These target proteins were prepared to be docked with main active ingredients analyzed earlier.

MCODE analysis is aimed to find clusters with highly interconnected regions in a network. In this study, MCODE analysis based on the above PPI network resulted in three cluster networks by plugins in Cytoscape software (Figure 5B-D and Supplementary file, Table S4). The higher score the modules got, the more meaningful modules in the PPI network was regarded as.

\section{Verification of Ligand-Receptor}

\section{Interaction}

Because of some missing receptor structures in the PDB website, AKT1 (id: 1 unq), EGF (id: 1epg), IL6 (id: 1alu), 


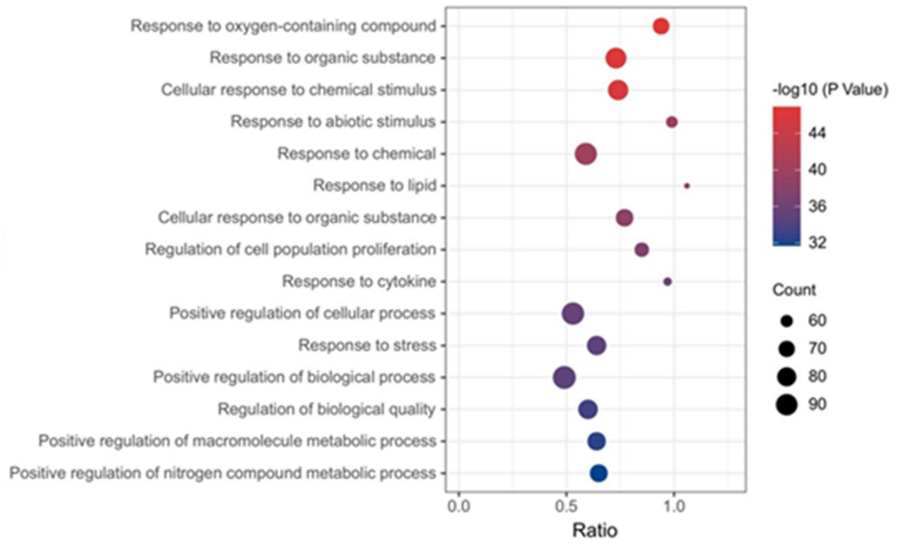

$A(B P)$

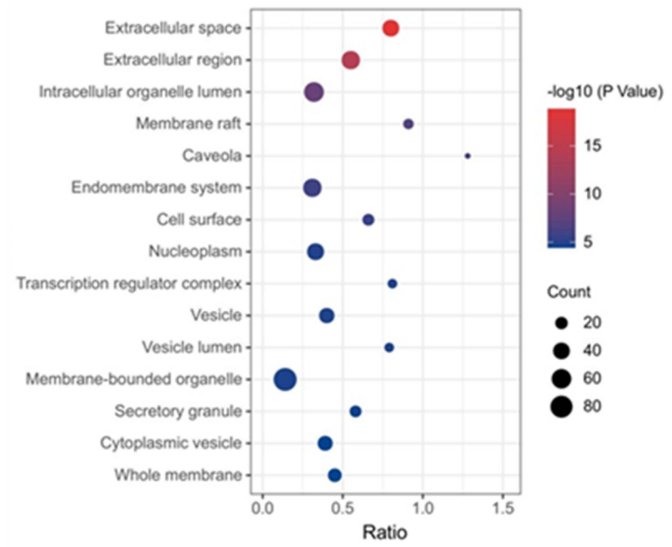

B (CC)

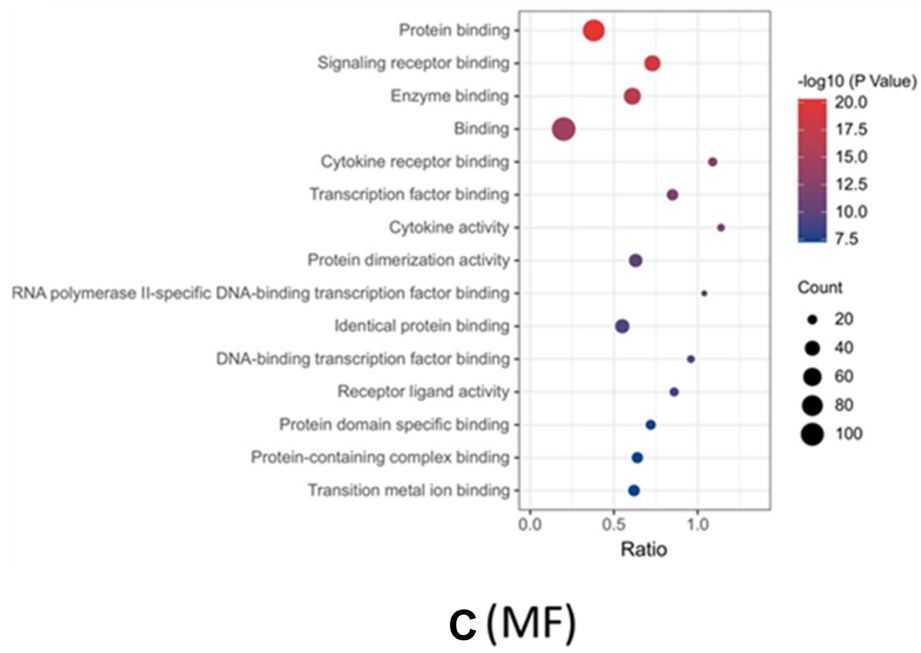

Figure 2 The GO enrichment analysis of genes of Li-Da-Qian mixture against glomerulonephritis, including three domains: biological process (A), cellular component (B), and molecular function (C). The enrichment ration, size and color of the dots represent the degree of GO enrichment analysis.

MAPK1 (id: 6slg), MMP9 (id: 5th6), TNF (id: 5uui), TP53 (id: 1aie) and VEGFA (id: 3qtk) were eventually selected based on the former conclusions. The docking ligands consisted of quercetin, luteolin, baicalein, but excluding $\beta$ amyrin acetate because relevant data is devoid in TCMSP The detailed docking procedures were seen in the "Materials and Methods" section. The whole results are shown in Table 3, Figure 6.1 and 2. Figure 6.1 indicated $3 \mathrm{D}$ interactions between ligands (usually assigning chemical compounds as ligands) and receptors (usually assigning proteins as ligands). Figure 6.2 indicates $2 \mathrm{D}$ interactions between compounds and targets. For example, Figure 6.2A indicates that baicalein formed an $\mathrm{H}$ bond conjugation with GLU 416, a $\pi$-H bond with ARG 424 and seven $\pi$ bonds in MMP9. The MAPK1-quercetin complex was stabilized by a $\pi-\mathrm{H}$ bond, six $\mathrm{H}$ bonds and eight $\pi$ bonds (Figure 6.2B). Quercetin interacted with VEGFA via six $\mathrm{H}$ bonds and three $\pi$ bonds on ASP56, LEU59, CYS54, GLY52, ASP27, CYS44 and CYS53 (Figure 6.2C). MAPK1 and luteolin were bound in GLU71, ASP167, GLN105, VAL39, MET108, ILE31, LEU156, ALA52, CYS166, LYS54 by two $\pi$-H bonds, five $\mathrm{H}$ bonds and seven $\pi$ bonds (Figure 6.2D). MMP9 and luteolin were conjugated by two $\pi$-H bonds, three $\pi$ bonds and four $\mathrm{H}$ bonds. The VEGFA-luteolin complex was combined by four $\mathrm{H}$ bonds on ASN55, ASP56 and CYS54, and four $\pi$ bonds on PHE29, ILE39, SER43 and CYS53 (Figure 6.2F). Interestingly, baicalein interacted with VEGFA by the same active sites as the interaction with luteolin except an H bond on GLY52 (Figure 6.2G). Quercetin also interacted with MMP9 by $\mathrm{H}$ bonds and $\pi$ bonds (Figure $6.2 \mathrm{H}$ ). Some other docking results were presented in Figure S3.1 and S3. 2. Among these docking results, the docking energy of baicalein with MMP9 $\left(-8.9 \mathrm{kcal} \cdot \mathrm{mol}^{-1}\right)$ was the lowest, 
Table 2 KEGG Pathway Analysis of Li-Da-Qian Mixture in Treating Glomerulonephritis

\begin{tabular}{|c|c|c|c|c|c|c|}
\hline Gene Set & Description & Size & Expect & Ratio & P Value & FDR \\
\hline hsa05200 & Pathways in cancer & 524 & 7.8553 & 6.1105 & 0 & 0 \\
\hline hsa05I63 & Human cytomegalovirus infection & 225 & 3.3730 & 8.3012 & 0 & 0 \\
\hline hsa05167 & Kaposi sarcoma-associated herpesvirus infection & 186 & 2.7883 & 9.3246 & 0 & 0 \\
\hline hsa0516I & Hepatitis B & 144 & 2.1587 & 13.897 & 0 & 0 \\
\hline hsa054I8 & Fluid shear stress and atherosclerosis & 138 & 2.0688 & 11.601 & 0 & 0 \\
\hline hsa04668 & TNF signaling pathway & 110 & 1.6490 & $13.34 \mid$ & 0 & 0 \\
\hline hsa05I42 & Chagas disease (American trypanosomiasis) & 102 & 1.5291 & 13.08 & 0 & 0 \\
\hline hsa04933 & AGE-RAGE signaling pathway in diabetic complications & 99 & $1.484 \mid$ & 20.888 & 0 & 0 \\
\hline hsa052I5 & Prostate cancer & 97 & $1.454 \mid$ & 13.754 & 0 & 0 \\
\hline hsa04657 & IL-I7 signaling pathway & 93 & 1.3942 & 16.497 & 0 & 0 \\
\hline hsa05I40 & Leishmaniasis & 74 & 1.1093 & 16.226 & 0 & 0 \\
\hline hsa05219 & Bladder cancer & 41 & 0.61463 & 26.032 & 0 & 0 \\
\hline hsa05222 & Small cell lung cancer & 92 & 1.3792 & $13.05 \mid$ & $8.88 E-16$ & $2.23 \mathrm{E}-14$ \\
\hline hsa04066 & HIF-I signaling pathway & 100 & $1.499 \mid$ & 12.007 & $3.89 \mathrm{E}-15$ & $9.05 \mathrm{E}-14$ \\
\hline hsa052I 2 & Pancreatic cancer & 75 & 1.1243 & $|4.23|$ & $8.66 \mathrm{E}-\mathrm{I} 5$ & $1.88 \mathrm{E}-13$ \\
\hline
\end{tabular}

indicating that the stability and binding ability of baicaleinMMP9 complex was higher compared with the other combinations. The above docking results suggested that glomerulonephritis may be alleviated by improving inflammation, reducing extracellular matrix deposition and repairing damaged blood vessels.

\section{Some Important Ingredients of Li-Da-Qian} Mixture Acts as a Vital Role in InflammationRelated Kidney Disease Model

Quercetin is widely studied in the field of nephrology. Quercetin protects against cisplatin-induced or LPSinduced acute kidney injury by inhibiting the inflammatory infiltration of macrophage. ${ }^{22,23}$ Quercetin also inhibited mesangial cell proliferation in the early stage of diabetic nephropathy in vivo and vitro. ${ }^{24,25}$ The research of luteolin in the field of kidney diseases is also very popular. Luteolin ameliorated ischaemic acute kidney injury in mice by blocking fructokinase. ${ }^{26} \mathrm{It}$ plays a protective role in cobalt-, ischemia-reperfusion-, LPS- and cisplatin-induced kidney injury as well. ${ }^{27-30}$ Baicalein protects kidney from the hurt by cisplatin, radiation, pristine and ischemia- reperfusion. ${ }^{31-34}$ All of the above ingredients present the anti-inflammatory and injury-relieving effects of kidney. Because research on baicalein treating LPSinduced acute kidney injury is less than luteolin and quercetin, we selected baicalein to validate its protective effects in C57BL/6J mice injected with $30 \mathrm{mg} / \mathrm{kg}$ LPS. 5 of 9 survived in the LPS and LPS $+10 \mathrm{mg} / \mathrm{kg}$ baicalein group. 6 of 9 survived in the LPS $+20 \mathrm{mg} / \mathrm{kg}$ group. None died in the other group without LPS injection, but one sample in $10 \mathrm{mg} / \mathrm{kg}$ baicalein was excluded because of haemolysis. In Figure 7A, the physiological and biochemical index of liver, kidney and heart was tested. The function of liver, kidney and heart after $24 \mathrm{~h}$ LPS treatment was significantly impaired as shown by increased serum AST, ALT, Cr, BUN, CK-MB, and LDH. Strikingly, both $10 \mathrm{mg} / \mathrm{kg}$ and $20 \mathrm{mg} / \mathrm{kg}$ baicalein treatment remarkably improved liver function as shown by reduced AST. There was also a significant decreased Cr between LPS group and LPS + $10 \mathrm{mg} / \mathrm{kg}$ baicalein group, which meant baicalein can improve renal function. Although there was no significant difference about LDH and CK-MB between LPS and LPS $+10 \mathrm{mg} / \mathrm{kg}$ baicalein group or LPS $+20 \mathrm{mg} / \mathrm{kg}$ baicalein group, a downtrend indicated that baicalein 


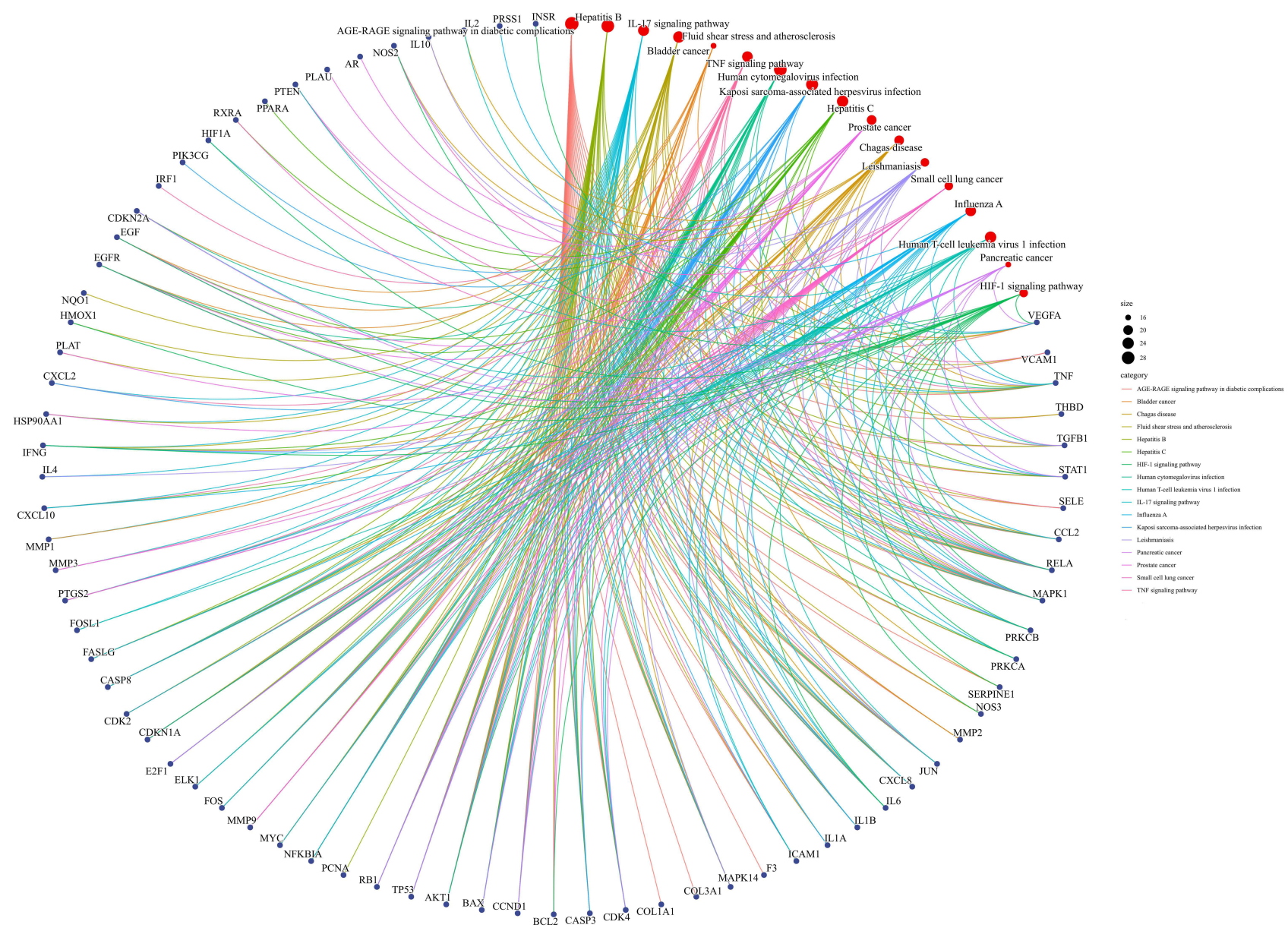

Figure 3 The KEGG analysis of genes of Li-Da-Qian mixture against glomerulonephritis. Different colors represent different pathways. The size of red dots represents amounts of enriched genes.

might markedly lower LDH or CK-MB when group numbers are expanded. Next, we examined mRNA levels of inflammatory indexes in renal cortex by qPCR. In Figure 7B, most inflammatory markers including IL6, IL18, iNOS, IL1 $\beta$ were significantly increased at $24 \mathrm{~h}$ after the injection of LPS; however, LPS did not affect TNF $\alpha$ levels. We found that $10 \mathrm{mg} /$ $\mathrm{kg}$ baicalein treatment significantly reduced IL6 and IL18 mRNA, interestingly, $10 \mathrm{mg} / \mathrm{kg}$ baicalein did not influence iNOS mRNA level while $20 \mathrm{mg} / \mathrm{kg}$ baicalein significantly decreased iNOS mRNA. Further, H \& E staining revealed that LPS induced hemorrhage in the interstitial tissue and infiltration in inflammatory cell. Treatment of LPS-injected mice with baicalein showed less hemorrhage and inflammatory infiltration vs LPSinjected mice with vehicle (Figure 7C).

\section{Li-Da-Qian Mixture Extract Regulates mRNA and Protein Expression in the LPS-Induced Glomerulonephritis Model}

With the development of single-cell sequencing, the occurrence and progress of many diseases usually attribute to two or more cell types. The former research of anti-inflammatory effects of luteolin and baicalein mostly focused on the macrophages, which exists in the blood system and is distributed in all organs. We aimed to study whether baicalein and luteolin can attenuate the inflammation induced by LPS in mesangial cells, which is specific in kidney tissue. In order to verify the effect of Li-Da-Qian mixture extract on inflammatory factors in mesangial cells, the mouse mesangial cells were pretreated with baicalein at $20 \mu \mathrm{M}$ or luteolin at $5 \mu \mathrm{M}$ for $2 \mathrm{~h}$, and then treated with 


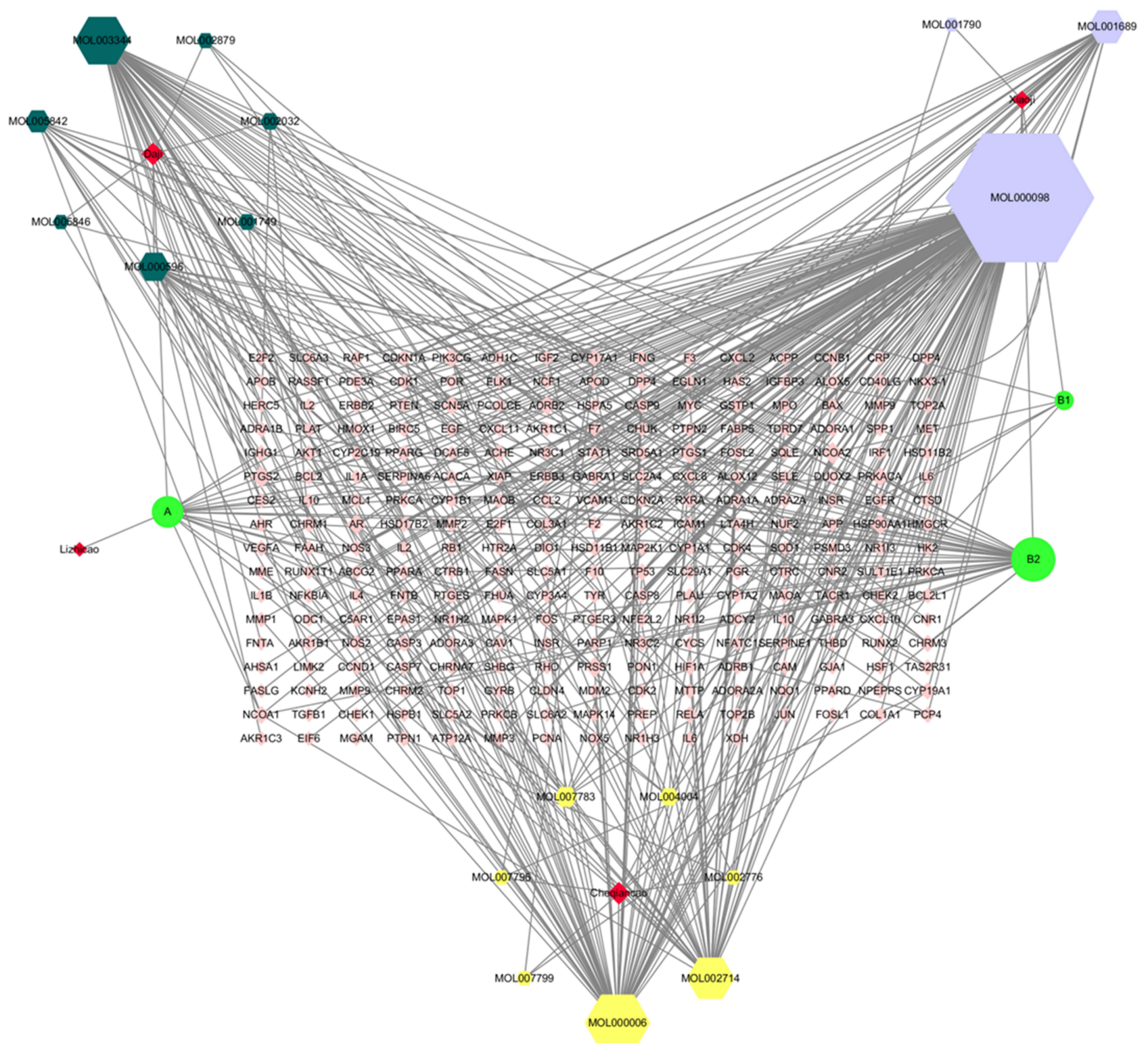

Figure 4 Drug targets network of Li-Da-Qian mixture. The potential target network of Li-Da-Qian is constructed using 20 compounds and 244 potential targets. Lines stand for the interactions between compounds and target nodes. A stands for MOL001735, which belongs to Daji, Cheqiancao and Lizhicao. BI stands for MOL000359, which belongs to Daji, Xiaoji and Cheqiancao. B2 stands for MOL000449, which belongs to Daji, Xiaoji and Cheqiancao.

$20 \mu \mathrm{g} / \mathrm{mL}$ LPS for another $24 \mathrm{~h}$. The baicalein concentration was based on the former literature, ${ }^{35}$ and the luteolin concentration was from CCK8 results (Figure S4). As presented in Figure $8 \mathrm{~A}-\mathrm{C}, \mathrm{E}, \mathrm{F}$, the mRNA and protein expression of iNOS in the LPS group was significantly higher than that in the regular group. At the same time, both baicalein and luteolin significantly decreased the expression of iNOS in mesangial cells treated with LPS $(\mathrm{P}<0.05)$, which indicated that active components in LiDa-Qian mixture were able to inhibit the expression of iNOS in mesangial cells. Figure $8 \mathrm{C}$ and $\mathrm{G}$ showed that
LPS also induced increased IL6 mRNA level in mesangial cells which can be ameliorated by baicalein or luteolin. However, TNF $\alpha$ and IL1 $\beta$ were not increased by LPS at $20 \mu \mathrm{g} / \mathrm{mL}$ (Figure $8 \mathrm{C}$ and $\mathrm{G}$ ), which may be associated with the concentration of LPS and the types of mesangial cells. Besides, baicalein and luteolin can significantly lower MMP2 mRNA and luteolin can elevate IL10 mRNA additionally (Figure 8D, G, H), and IL10 is usually regarded as an anti-glomerulonephritis marker. ${ }^{36,37}$

NF- $\mathrm{B}$ Signaling, Jak/Stat Signaling (IL-6 Receptor Family), P53 signaling and NLRP3 inflammasome 


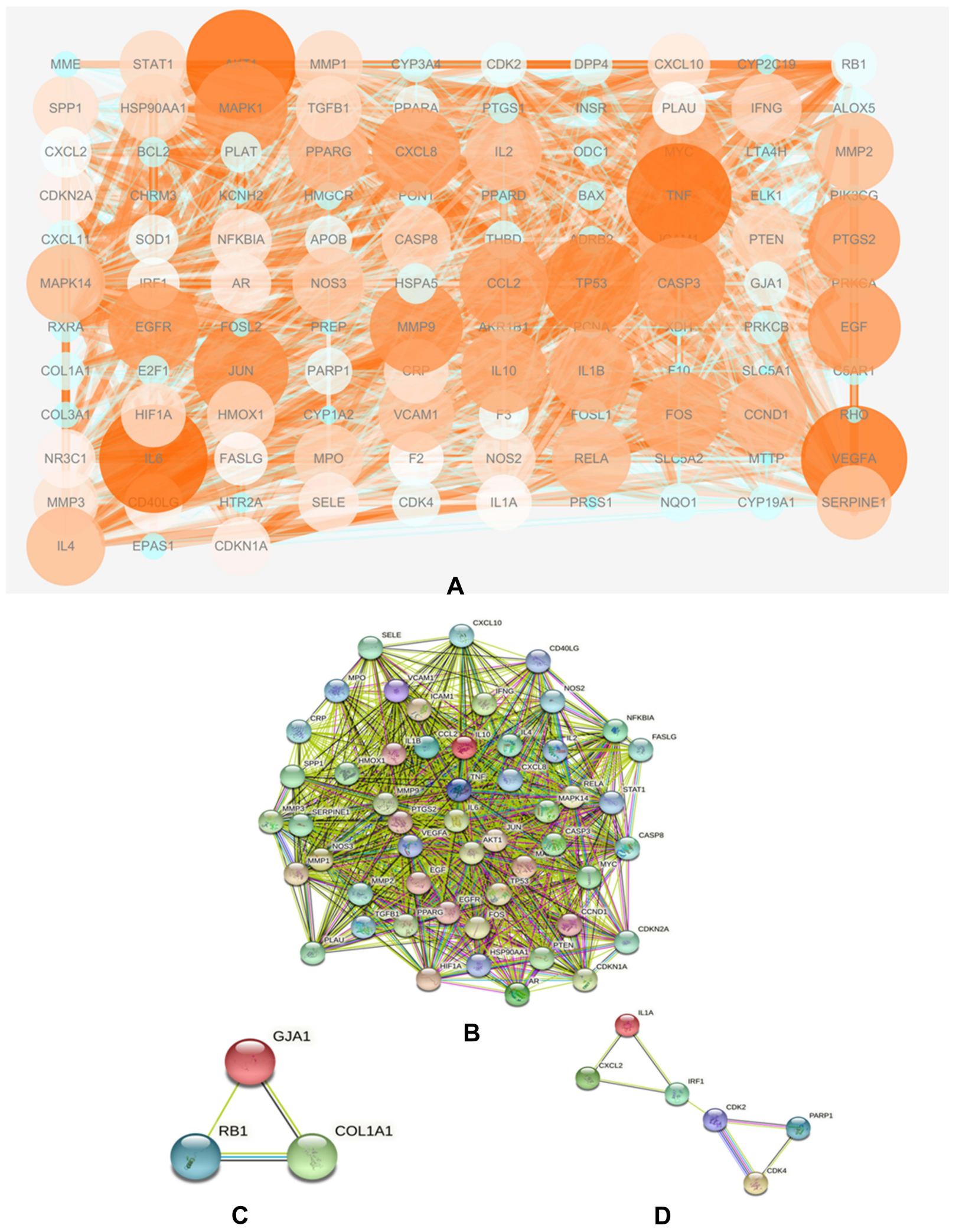

Figure 5 (A) PPI network of Li-Da-Qian mixture-glomerulonephritis genes. The network (A) contains I I 3 nodes and I 967 edges. Core genes have a higher degree and are positively correlated with node size and color depth, including, IL-6, VEGFA, EGF, MAPKI, MMP9, TP53, TNF, and (B-D) MCODE analysis of PPI network in (A). Cluster 5 (B) includes 52 nodes and 1068 edges, Cluster 5 (C) includes 3 nodes and 3 edges and Cluster5 (D) includes 6 nodes and 7 edges. 
Table 3 Docking Results of Three Bioactive Ingredients from Li-Da-Qian Mixture for Glomerulonephritis Targets

\begin{tabular}{|c|c|c|c|c|c|c|c|c|c|}
\hline \multirow[t]{2}{*}{ Ingredients } & \multirow[t]{2}{*}{ Structure } & \multicolumn{8}{|c|}{ Binding Energy/(kcal/mol) } \\
\hline & & AKTI & IL6 & VEGFA & TNF & TP53 & EGF & MAPKI & MMPQ \\
\hline Quercetin & & -6.4 & -6.4 & -8.2 & -6.8 & -6.5 & -5.9 & -8.3 & -7.6 \\
\hline Baicalein & & -6.8 & I & -8.1 & I & -6.7 & -5.9 & 1 & -8.9 \\
\hline luteolin & & -6.2 & -6.5 & -8.2 & -6.8 & -6.6 & -5.9 & -8.3 & -8.3 \\
\hline
\end{tabular}

activation are closely related to inflammatory disorders. In this study, some key proteins in the above signaling were detected after considering docking receptor proteins. As shown in Figure 8A and B, COX2 was greatly induced by LPS, but cannot be blunted by baicalein. Previous study has illustrated that the anti-apoptosis and anti-inflammatory effects of baicalein related to glomerulonephritis are embodied in an increase of Nrf2 and decreased levels of reactive oxygen species and suppressed the phosphorylation of NF- $\mathrm{KB}$ in myeloid-derived suppressor cells (MDSCs). ${ }^{32}$ Therefore, we detected Nrf2 protein level and no significant results were found in mesangial cells. Docking results showed baicalein interacted with TP53, so we examined phosphorylated P53 and total P53 in LPS stimulated-mesangial cells. The Western blot data showed that LPS significantly decreased the total P53 protein level (Figure $8 \mathrm{~A}$ and B). Although baicalein was unable to reverse the decline of the total form of P53, it brought an increase in the phosphorylation form of P53 surrounding Ser15 of human P53 (equal to Ser18 of mouse P53), which stabilized P53 and may alleviate para-inflammation and systemic inflammation. ${ }^{38-41}$ As presented in Figure 8E and F, NLRP3 was significantly blunted after luteolin treatment, which was greatly induced by LPS. Interestingly, luteolin is a potent nuclear factor E2-related factor 2 (Nrf2) inhibitor or activator in the different disease states, ${ }^{42-44}$ and Nrf2 is thought to be a major regulator of the antioxidant response. The results in Figure 8E and $\mathrm{F}$ showed that Nrf2 had a decreased trend between control group and LPS group, and was activated by luteolin.

\section{Li-Da-Qian Mixture Extract Regulates TGF $\beta /$ Smad Signaling in the TGF $\beta$ I- Induced mPTC Model}

As TGF $\beta /$ Smad signaling pathway is one of the most important pathways in glomerulonephritis development, we treated mPTC with baicalein or luteolin. As shown in Figure $9 \mathrm{~A}$ and $\mathrm{B}$, baicalein treatment profoundly prevented the fibrosis induced by TGF $\beta 1$. Baicalein lowers both phosphorylation and total form of $\mathrm{Smad} 2 / 3$ protein. These findings were supported by the results of qPCR, which demonstrated that baicalein significantly decreased the up-regulation of fibrosis markers, including FN, $\alpha$ SMA and collagen I (Figure 9C). In Figure 9D and E, luteolin also inhibited the high expression of fibronectin stimulated by TGF $\beta 1$. Unlike baicalein, luteolin affects the activation of $\operatorname{Smad} 2 / 3$ without changing the level of total $\operatorname{Smad} 2 / 3$. The mRNA levels of FN, $\alpha$-SMA and collagen I were reduced in the presence of luteolin when the mPTCs were challenged with TGF- $\beta 1$ (Figure 9F).

\section{Discussion}

Network pharmacology, also called integrated Systems Pharmacology, is a rational approach to the analysis of underlying molecular biological mechanism of TCM in pathological process of different kinds of diseases, and especially in chronic disease, such as glomerulonephritis. ${ }^{45}$ Although Li-Da-Qian mixture has been used clinically for many years and has a good effect, the mechanism of action is never explored. Therefore, network pharmacology was firstly performed to reveal the mechanism of Li-Da-Qian 


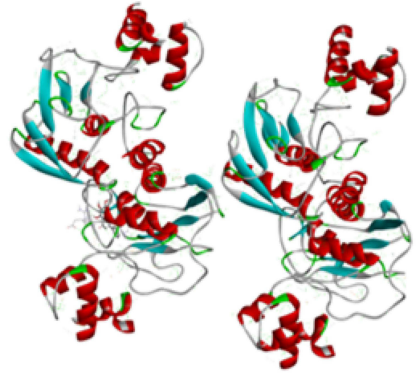

A

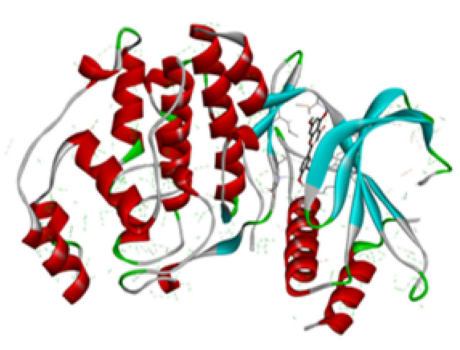

D

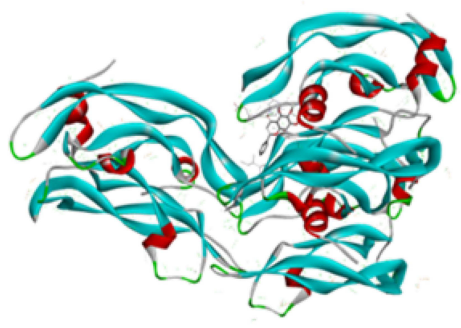

G

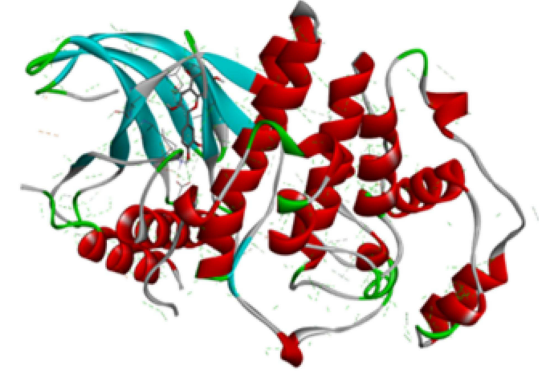

B

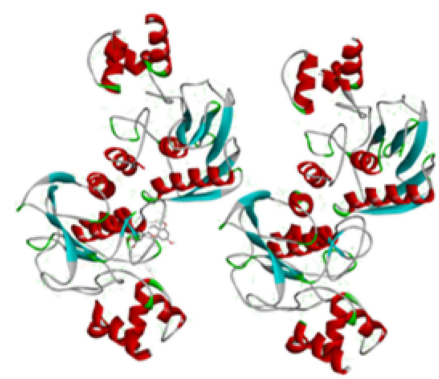

E

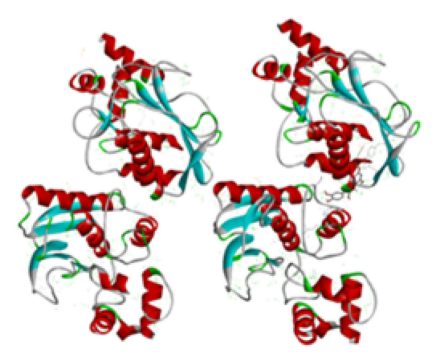

H

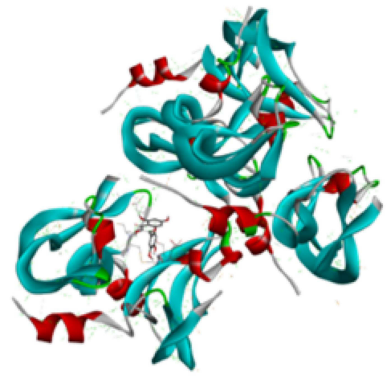

C

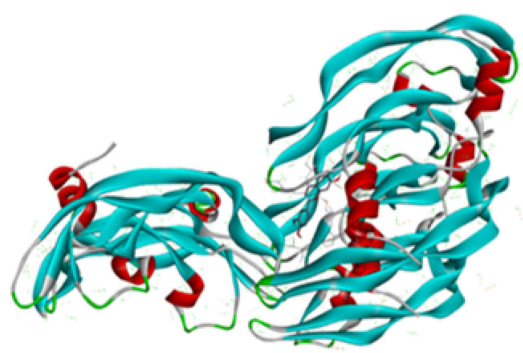

$\mathbf{F}$

Figure 6 Continue.

mixture treating glomerulonephritis in this study. By integrating all the targets of Li-Da-Qian mixture and glomerulonephritis, 113 targets related to glomerulonephritis were identified, such as IL6, AKT1, VEGFA, TNF, TP53, EGF, MAPK1, MMP9, etc. KEGG analysis revealed that "Fluid shear stress and atherosclerosis", "TNF signaling pathway", "IL-17 signaling pathway", "AGE-RAGE signaling pathway in diabetic complications" and "HIF-1 signaling pathway" were related to the mechanism of Li-Da-Qian mixture in the treatment of glomerulonephritis. The typical KEGG results highlighted the direct effect of Li-Da-Qian mixture on glomerulonephritis, and also showed "multiply compounds, multiply targets, multiply pathways" potential mechanisms. Molecular docking provided guarantee of network pharmacology. The docking results indeed proved that
Li-Da-Qian mixture may target some key proteins in the inflammation pathway.

Quercetin, luteolin and baicalein were eventually recognized as the most important active ingredients of Li-Da-Qian mixture in the treatment of glomerulonephritis. Quercetin (Sophoretin) is a plant-based chemical, which is considered to be a flavonoid compound derived from fruits, vegetables, leaves and grains. It may also be used as an ingredient in supplements, drinks and foods. Quercetin is a PI3K inhibitor with IC50 of $2.4-5.4 \mu \mathrm{m}$ and also a stimulator of recombinant SIRT $1 .^{46,47}$ It can effectively disable PI3K and Src kinases, moderately inhibit Akt1/2, and slightly affect PKC, p38 and ERK1/2. ${ }^{46}$ Previous studies have shown that quercetin lowers inflammation and apoptosis by 


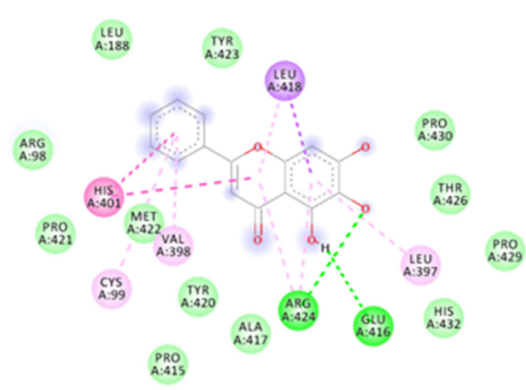

A

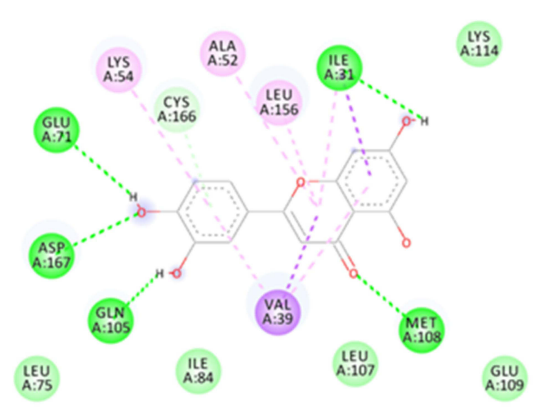

D

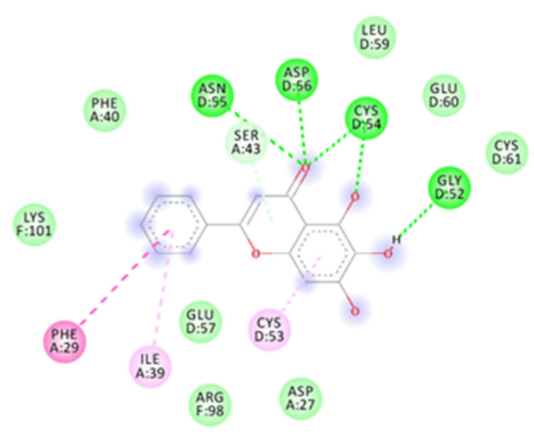

G

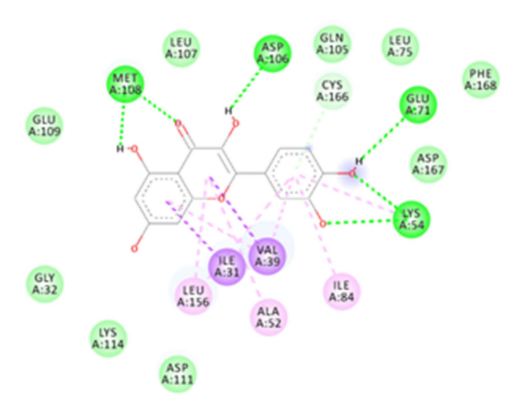

B

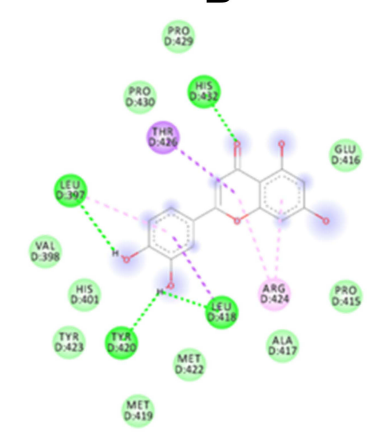

E

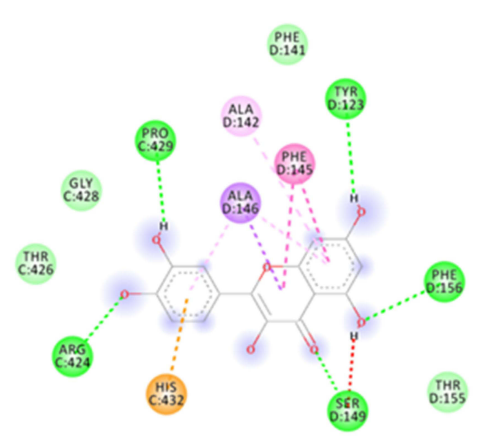

H

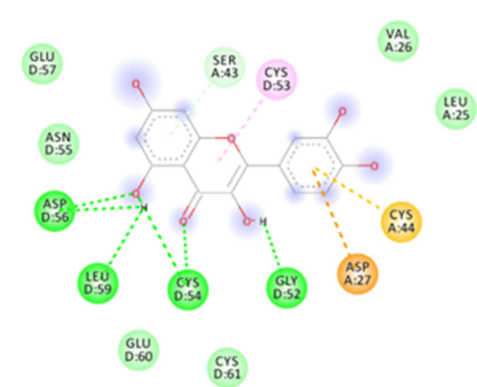

C

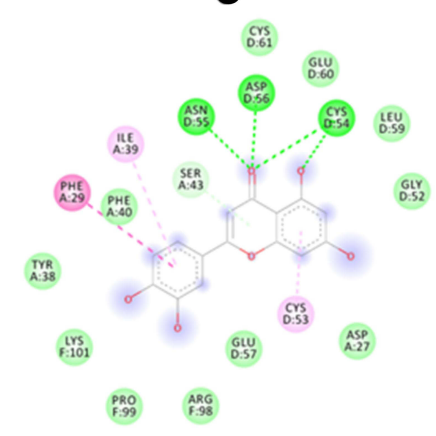

$\mathbf{F}$

\section{Interactions}

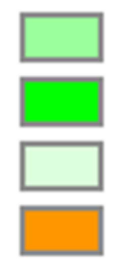

$v$ an der "laals

Conventional Hydrogen Bond

Carbon Hydrogen Bond

Pi-Cation

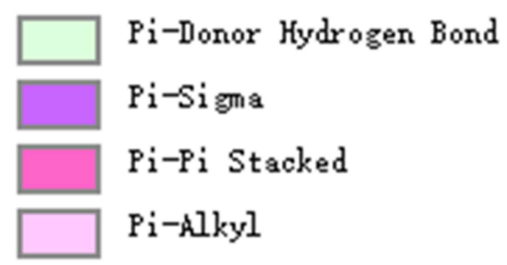

Figure 6 I. Virtual 3D docking of bioactive ingredients from Li-Da-Qian mixture for glomerulonephritis targets. The molecular docking of baicalein, luteolin and quercetin with MMP9 (A, E, H), MAPKI (/, D, B) and VEGFA (G, F, C) is shown. 2. Virtual 2D docking of bioactive ingredients from Li-Da-Qian mixture for glomerulonephritis targets. The molecular docking of baicalein, luteolin and quercetin with MMP9 (A, E, H), MAPKI (I, D, B) and VEGFA (G, F, C) is shown.

downregulating TNF- $\alpha$, IL-6, TGF- $\beta 1$ and Bax in the pristane-induced Lupus nephritis (LN) mice model. ${ }^{48}$ Meanwhile, quercetin regulates oxidative stress by increasing superoxide dismutase 1 (SOD1) and catalase
(CAT) ${ }^{48,49}$ Quercetin probably exerts antioxidant effects on NF- $\mathrm{KB}$ signaling in RAW264.7 cells. ${ }^{50}$ Luteolin, a flavonoid compound found in Terminalia chebula, is a non-selective PDE inhibitor with IC50 less than $1 \mu \mathrm{m}$, 
A
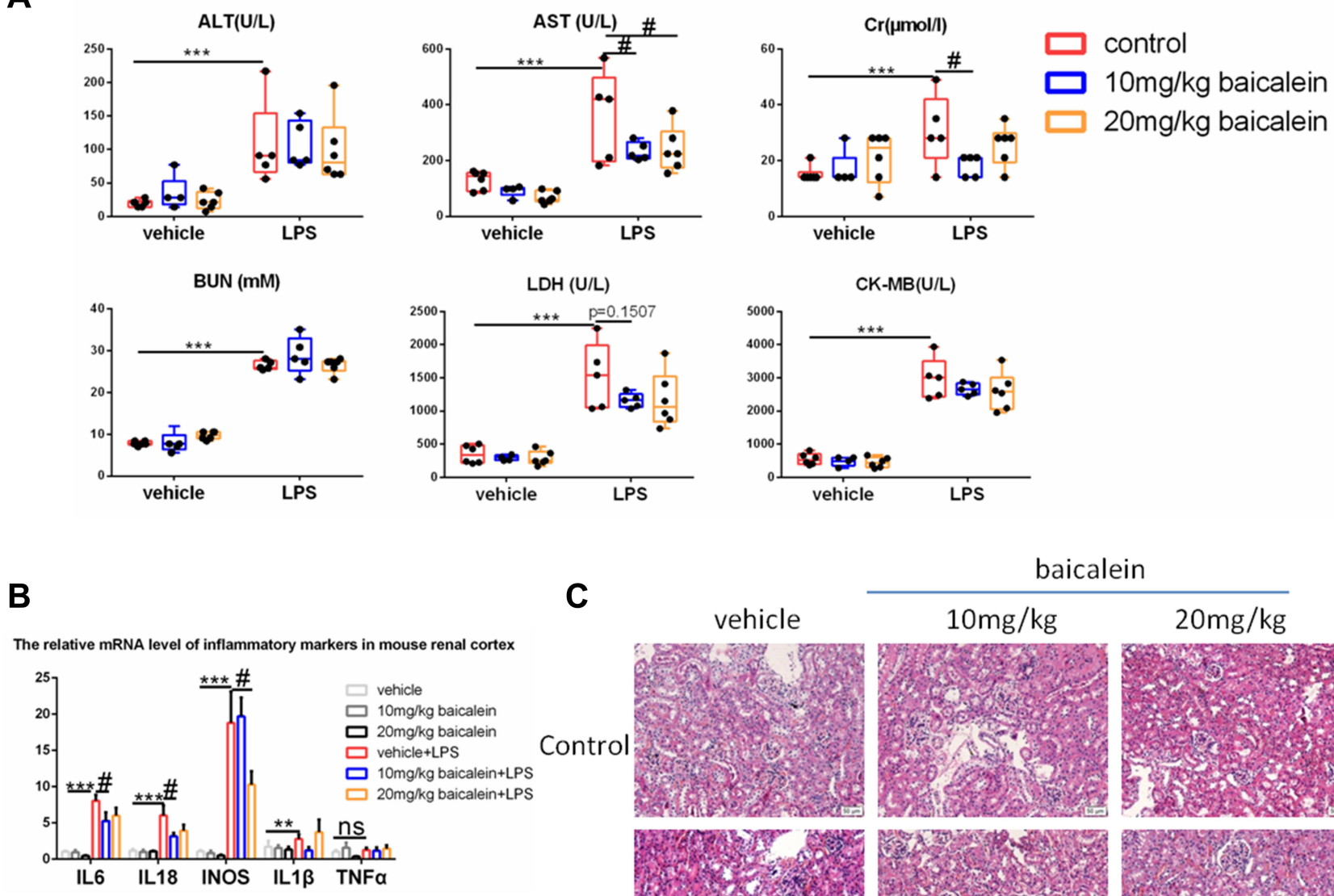

LPS
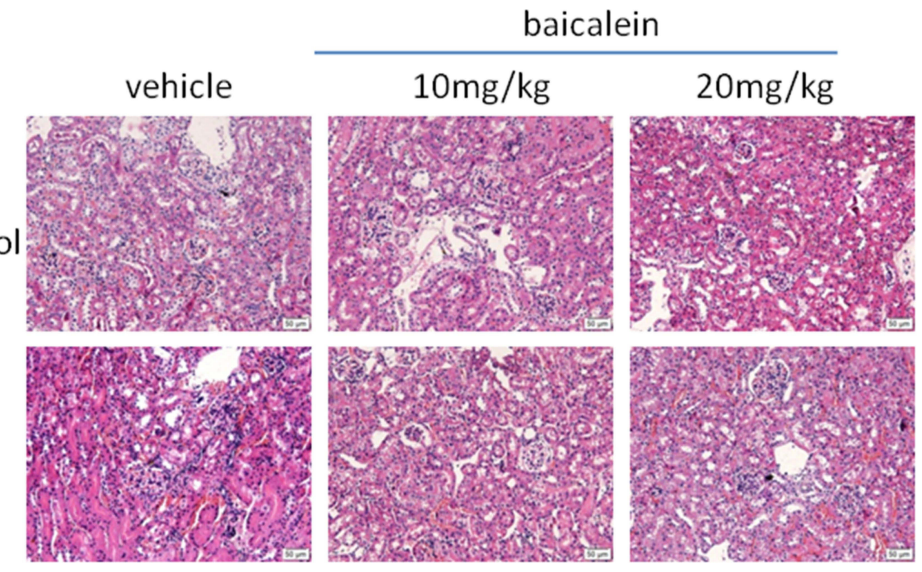

Figure 7 Baicalein treatment protected from LPS-induced acute kidney injury. (A) Serum biochemical levels in mice $(n=5-6)$ treated with different doses of baicalein after 24h LPS administration. (B) The inflammatory factors in the renal cortex of LPS-induced acute kidney injury model with or without different doses of baicalein ( $\mathrm{n}=5-6)$. (C) Representative images of hematoxylin and eosin staining (magnification: $\times 200$, scalebar: $50 \mu \mathrm{m}$ ) of kidneys after $24 \mathrm{~h}$ LPS administration. ${ }^{* *} \mathrm{p}<0.01$, ${ }^{* * *} \mathrm{p}<0.00 \mathrm{I}$ versus the control group, $\# \mathrm{p}<0.05$ versus the LPS group.

which inhibits TNF- $\alpha$ production stimulated by LPS and LPS-induced phosphorylation of Akt and rescues LPSinduced phosphorylation of NF-kappa-B inhibitor alpha (NFKBIA) and secondary degradation in RAW264.7 cell. ${ }^{51,52}$ Luteolin can also target IL18 and IL1 $\alpha$ to protect from inflammation infiltration in nonalcoholic steatohepatitis. ${ }^{53}$ However, we cannot see a significant decline in IL18 mRNA level by luteolin in mesangial cells, which may be caused by different effects of luteolin on different cell types or different concentration of luteolin. Experiments in vivo have proved that luteolin is an effective nephroprotective agent likely to reduce platinum accumulation in the kidneys and ameliorate cisplatin-induced nephrotoxicity. ${ }^{30}$ Baicalein is an inhibitor of cytochrome P450 2C9, prolyl oligopeptidase, 12-lipoxygenase and xanthine oxidase. ${ }^{54-57}$ Our results in vivo and vitro showed baicalein effectively decreased pro-inflammatory factors, especially iNOS, IL6. On the basis of these findings, we hypothesize that Li-Da-Qian mixture may exert anti-glomerulonephritis effects via different pathways in different kinds of cells including macrophages, myeloid-derived suppressor cells and mesangial cells (summarized in Figure 10).

In this study, network pharmacology was fully used to focus on the potential protective effect of Li-Da-Qian mixture, and this effect was visualized by molecular docking and experiments for verification. At the same time, we performed experiments in vivo and vitro to verify our findings. Although some docking results were not consistent in mesangial cells, some literature results seemed to verify our docking results in other cell lines. Thus, it is necessary for researchers to design more experiments to 
A mouse mesangial cells

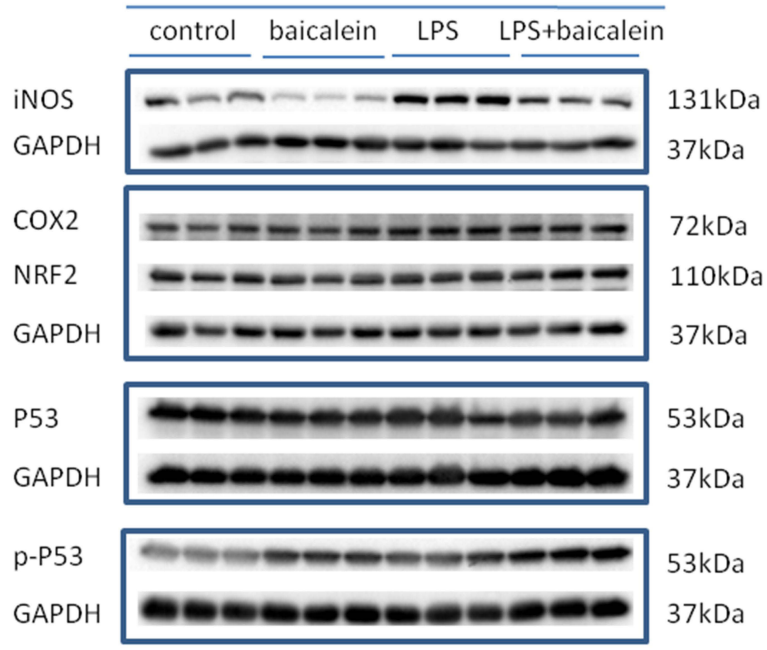

C The mRNA level of inflammatory mediators in mesangial cells

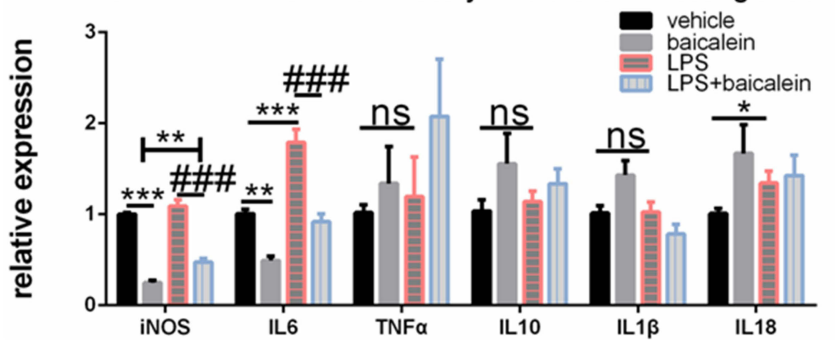

$\mathbf{E}$ mouse mesangial cells

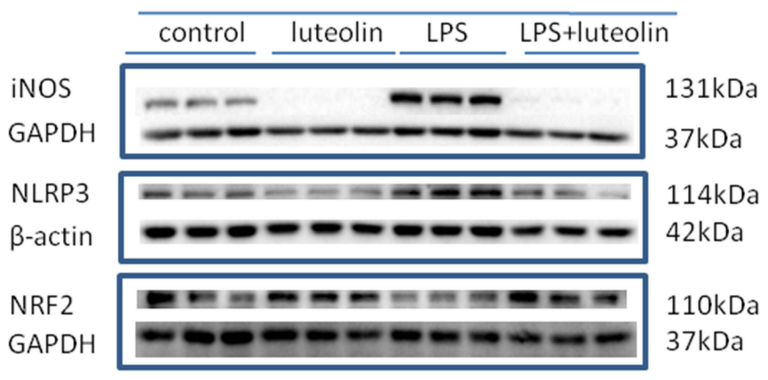

B

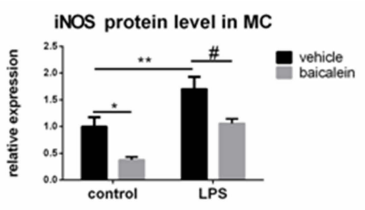

COX2 protein level in MC
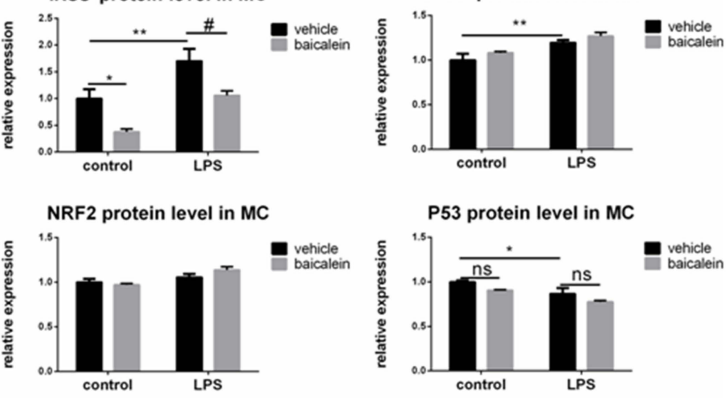

p-P53 protein level in MC
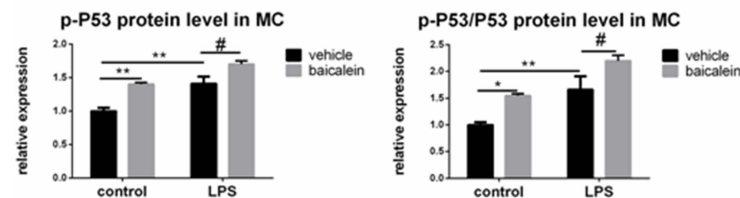

D

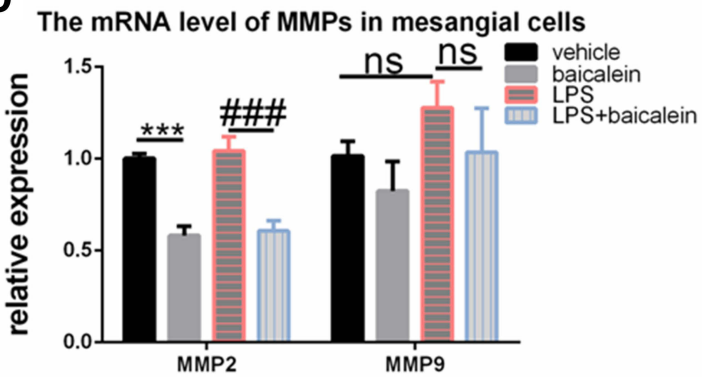

$\mathbf{F}$
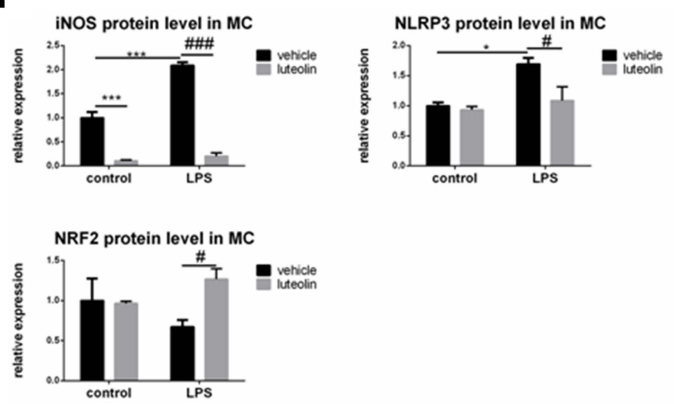

G The mRNA level of inflammatory mediators in mesangial cells $\mathbf{H}$
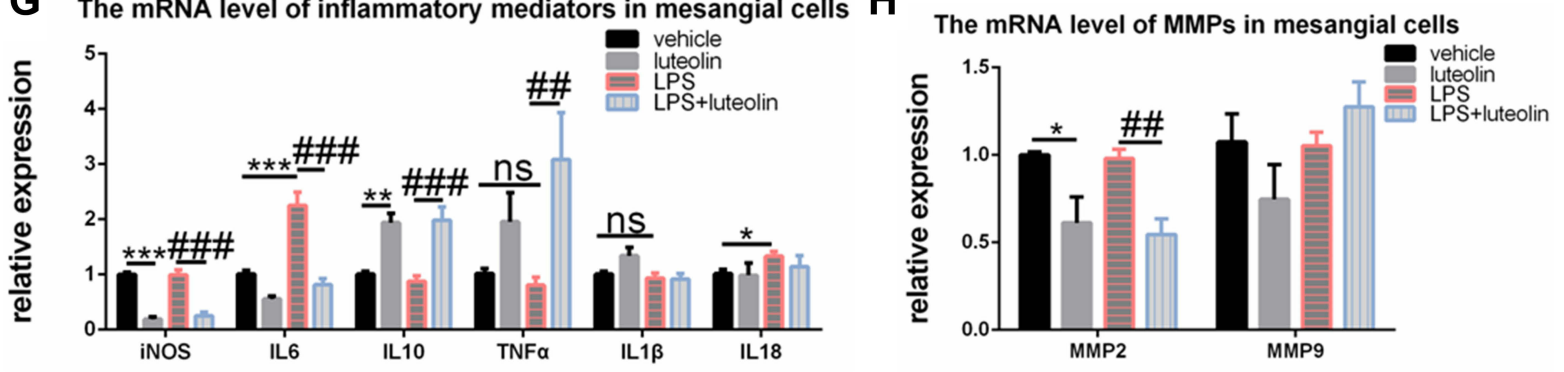

Figure 8 Effects of baicalein and luteolin treatment for LPS-induced glomerulonephritis by Western blotting and qPCR. Cells were grown on 6-well or I2-well plates until $60-70 \%$ confluence, pretreated with baicalein or luteolin for $2 \mathrm{~h}$, and sequentially stimulated with LPS $(20 \mu \mathrm{g} / \mathrm{mL})$ for another $24 \mathrm{~h}$. Western blotting results for baicalein therapeutic effect $(\mathbf{A})$, densitometric analysis $(\mathbf{B})$ and qPCR results for baicalein therapeutic effect (C and $\mathbf{D})$. Western blotting results for luteolin therapeutic effect (E), densitometric analysis $(\mathbf{F})$ and $q P C R$ results for luteolin therapeutic effect $(\mathbf{G}$ and $\mathbf{H})$. Protein results are shown as mean \pm SEM $(n=3)$ and mRNA results are displayed as mean \pm SEM $(n=6) .{ }^{*} p<0.05,{ }^{*} p<0.01,{ }^{* * *} p<0.001$ versus the control group, ${ }^{\#} p<0.05,{ }^{m} p<0.01,{ }^{\# \#} p<0.001$ versus the LPS group. 
A

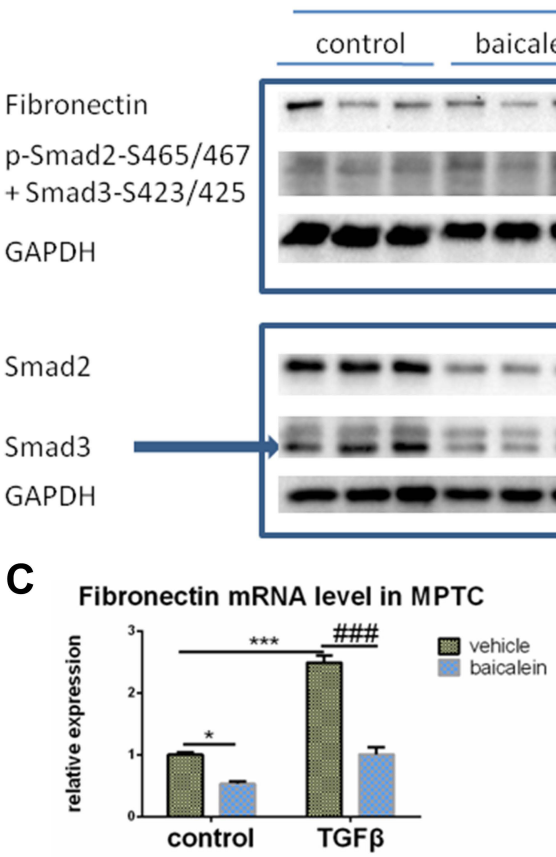

mouse proximal tubular cells

\section{TGF $\beta \quad$ TGF $\beta$ +baicalein \\ ein}

B

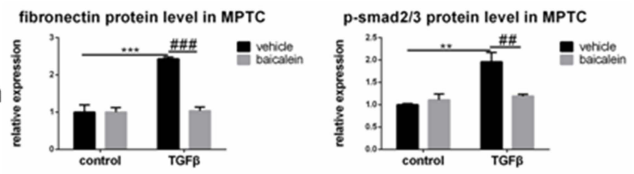

$60 \mathrm{kDa}$

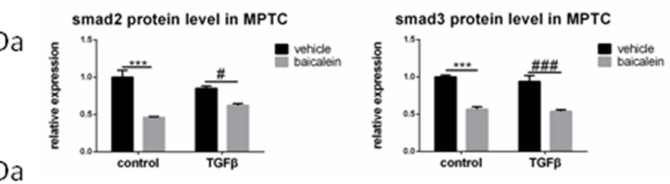

$60 \mathrm{kDa}$

$55 \mathrm{kDa}$

$37 \mathrm{kDa}$
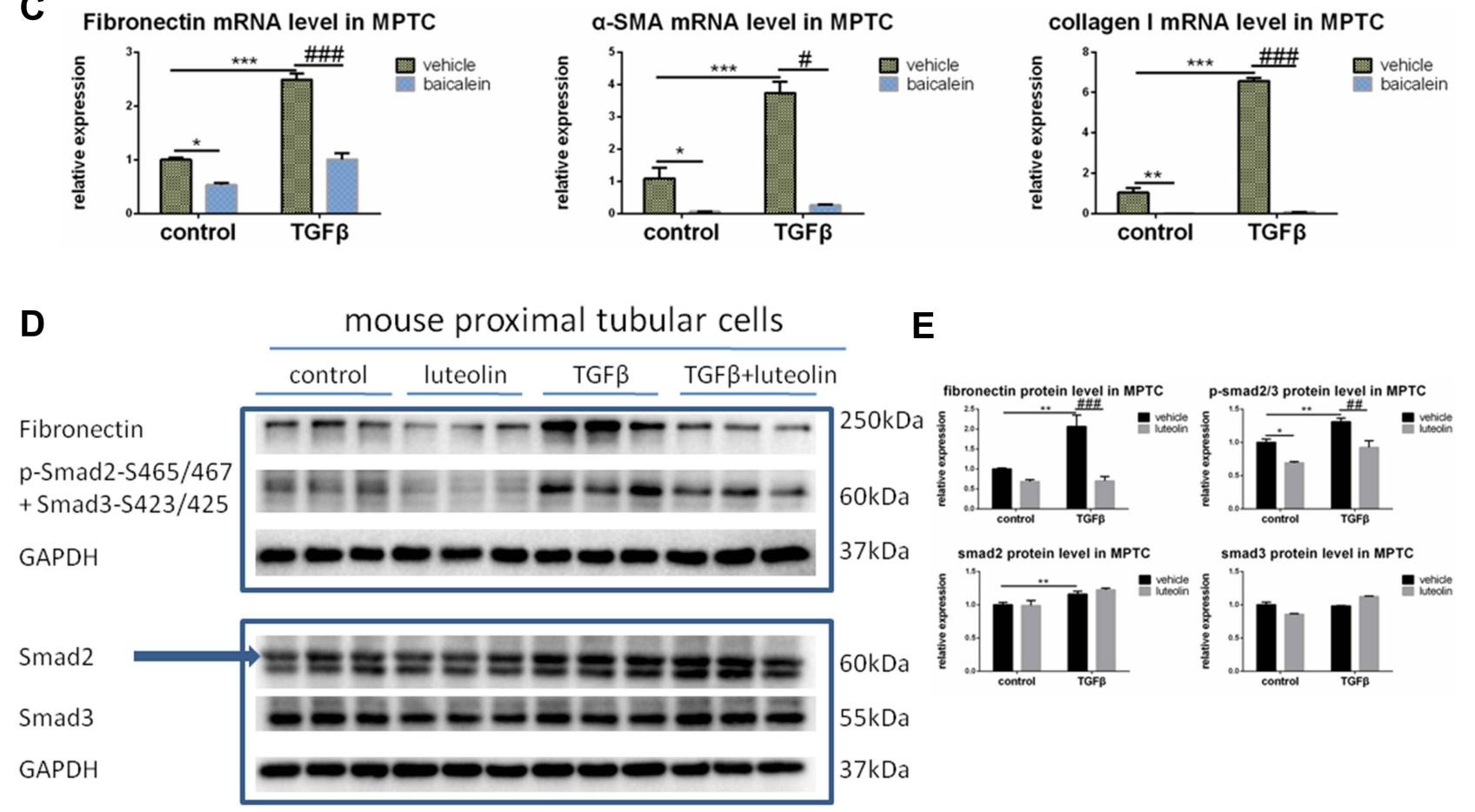

F

Fibronectin mRNA level in MPTC

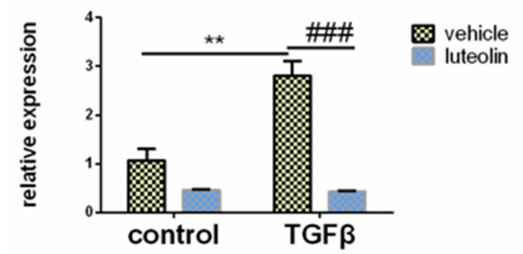

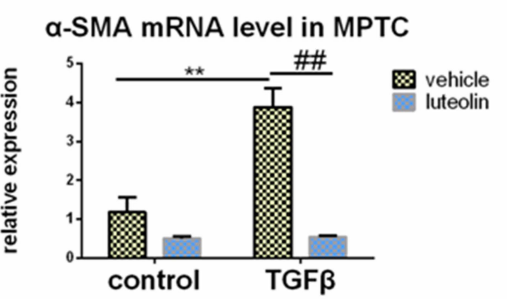

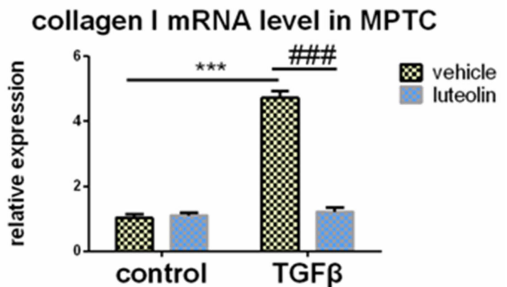

Figure 9 Effects of baicalein and luteolin treatment for TGF $\beta$ I-induced renal fibrosis by Western blotting and qPCR. Cells were grown on 6-well or I2-well plates until 60-70\% confluence, pretreated with baicalein or luteolin for $2 \mathrm{~h}$, and sequentially stimulated with TGF $\beta$ I (IOng/mL) for another $24 \mathrm{~h}$. Western blotting results for baicalein therapeutic effect $(\mathbf{A})$, densitometric analysis (B) and qPCR results for baicalein therapeutic effect (C). Western blotting results for luteolin therapeutic effect (D), densitometric analysis $(\mathbf{E})$ and $\mathrm{qPCR}$ results for luteolin therapeutic effect $(\mathbf{F})$. Protein and mRNA results are displayed as mean $\pm \mathrm{SEM}\left(\mathrm{n}=3\right.$ ). ${ }^{*} \mathrm{p}<0.05$, $* * \mathrm{p}<0.0 \mathrm{I}$, *** $\mathrm{p}<$ 0.001 versus the control group, ${ }^{\#} p<0.05,{ }^{\# \#} p<0.01,{ }^{\# \#} p<0.001$ versus the TGF $\beta$ I group.

study the complex regulation among different cells in kidney. Additionally, it is meaningful to explore whether Li-Da-Qian mixture can treat other kidney diseases.

\section{Conclusion}

TCM has provided enough information about alleviation of glomerulonephritis progression. In our study, core 


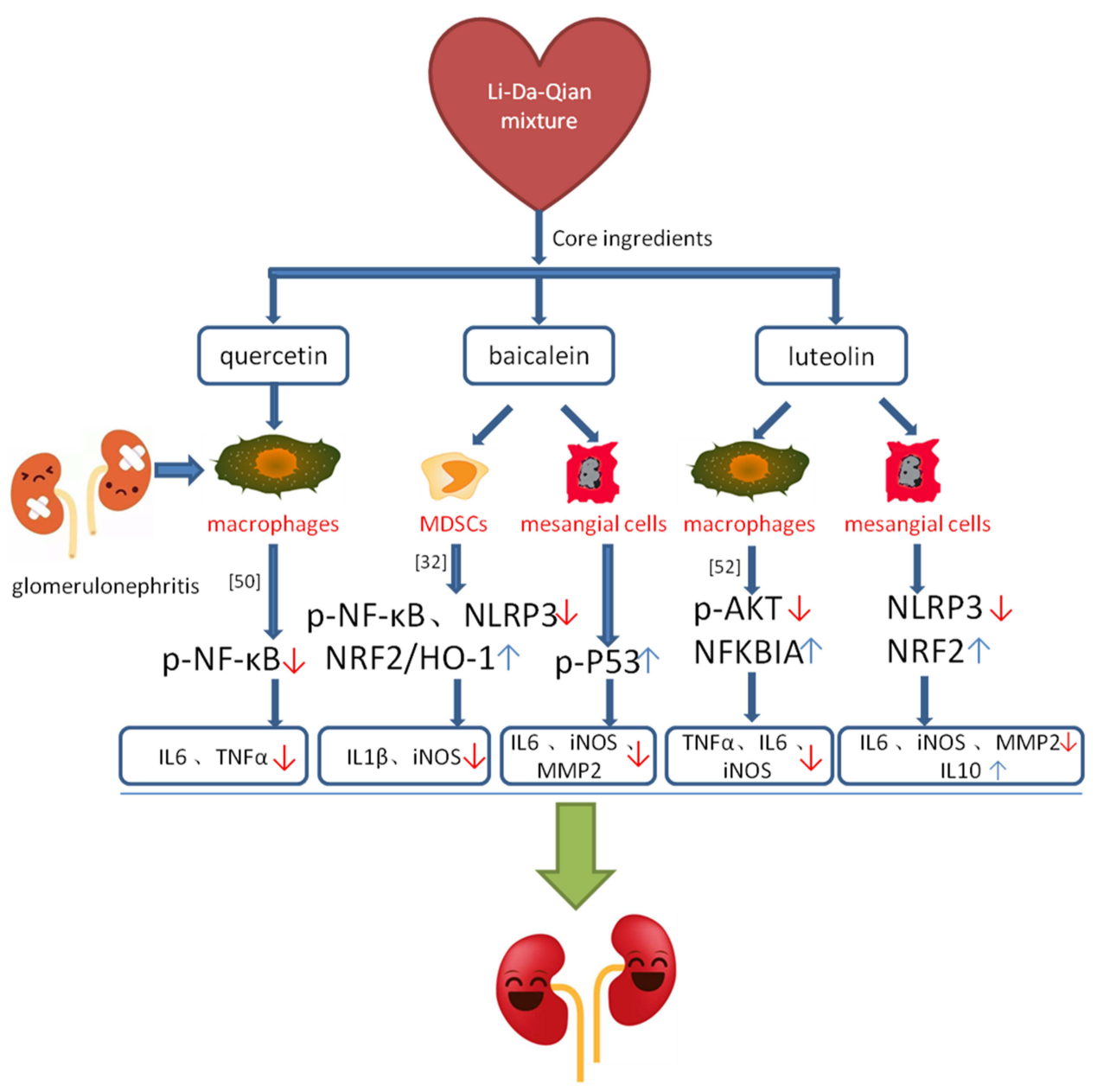

Figure 10 Possible mechanisms of Li-Da-Qian mixture core compounds on glomerulonephritis.

components of Li-Da-Qian mixture can significantly decrease IL6, iNOS and MMP2 expression and increase IL10 expression in LPS-induced glomerulonephritis. Summarizing some conclusions from other researchers, the ingredients of Li-Da-Qian mixture may also lower other cytokines, such as IL1, TNF, MMP9, etc, which were identified by the pharmacological network as well. The reliability of these results deserves more experiments to validate.

\section{Acknowledgments}

The authors thanks Dr Jun Chen for providing iNOS antibody; Bingyu Yang for providing NLRP3, COX2 antibody; and Shuang $\mathrm{Xu}$ for lending $\mathrm{p}-\mathrm{Smad} 2 / 3$ and total Smad2/3 antibody.

\section{Funding}

This work was supported by the fellowship of China Postdoctoral Science Foundation (2020M681674) and
National Natural Science Foundation of China (821070733).

\section{Disclosure}

The authors declare no duality of interest associated with this manuscript.

\section{References}

1. Cook HT, Pickering MC. in Heptinstall's Pathology of the Kidney 7th edn Vol. 1 Ch. 9 (eds Jennette, J. C. et al.) 341-366 (Wolters Kluwer, 2014).

2. Phillips TM, Fadia M, Lea-Henry TN, et al. MMP2 and MMP9 associate with crescentic glomerulonephritis. Clin Kidney J. 2017;10 (2):215-220. doi:10.1093/ckj/sfw111

3. Bauvois B, Mothu N, Nguyen J, et al. Specific changes in plasma concentrations of matrix metalloproteinase- 2 and -9 , TIMP- 1 and TGF-beta1 in patients with distinct types of primary glomerulonephritis. Nephrol Dial Transplant. 2007;22(4):1115-1122. doi:10. 1093/ndt/gfl743

4. Vira H, Pradhan V, Umare V, et al. Role of MMP-7 in the pathogenesis of systemic lupus erythematosus (SLE). Lupus. 2017;26(9):937-943. doi: $10.1177 / 0961203316682855$ 
5. Satoskar AA, Nadasdy T, Silva F. in Heptinstall's Pathology of the Kidney 7th edn Vol. 1 Ch. 10 (eds Jennette, J. C. et al.) (Wolters Kluwer, 2014).

6. World Health Organization. A Review of the Technical Basis for the Control of Conditions Associated with GAS Infections. WHO; 2005.

7. Satoskar AA, Parikh SV, Nadasdy T. Epidemiology, pathogenesis, treatment and outcomes of infection-associated glomerulonephritis. Nat Rev Nephrol. 2020;16(1):32-50. doi:10.1038/s41581-019-0178-8

8. Srivastava A, Cai X, Mehta R, et al. Hospitalization trajectories and risks of ESKD and death in individuals with CKD. Kidney Int Rep. 2021;6(6):1592-1602. doi:10.1016/j.ekir.2021.03.883

9. Zhu C, Huang S, Ding G, et al. Protective effects of Huang Qi Huai granules on Adriamycin nephrosis in rats. Pediatr Nephrol. 2011;26 (6):905-913. doi:10.1007/s00467-011-1808-y

10. Duan SB, Pan P, Xu Q, et al. Preliminary study of Huai Qi Huang granules delay the development of primary glomerular diseases in human. Ren Fail. 2014;36(9):1407-1410. doi:10.3109/0886022X.20 14.952746

11. Yu X, Meng X, Xu M, et al. Celastrol ameliorates cisplatin nephrotoxicity by inhibiting NF-kappaB and improving mitochondrial function. EBioMedicine. 2018;36:266-280. doi:10.1016/j.ebiom.2018.09. 031

12. Ma YG. Observation of Li-Da-Qian Mixture in Treating Children with Acute Nephritis. Jiangsu J Trad Chinese Med. 1989;(07). Available from: https://kns.cnki.net $/ \mathrm{kcms} /$ detail/detail.aspx?dbcode $=$ CJFD\&dbname $=$ CJFD8589\&filename $=J S Z Y 198907007 \&$ uniplatform $=$ NZKPT\& $=$ S Z l c F W x l j n 5 W j u f p r G z Y b W H r D j r W K C 01 kn FsAxarOlevt9DMj8yL3VVLnlp0XI0. Accessed November 26, 2021.

13. Lee WY, Lee CY, Kim YS, et al. The methodological trends of traditional herbal medicine employing network pharmacology. Biomolecules. 2019;9(8). doi:10.3390/biom9080362

14. Ru J, Li P, Wang J, et al. TCMSP: a database of systems pharmacology for drug discovery from herbal medicines. $J$ Cheminform. 2014;6:13. doi:10.1186/1758-2946-6-13

15. Huang $\mathrm{C}$, Zheng $\mathrm{C}$, Li $\mathrm{Y}$, et al. Systems pharmacology in drug discovery and therapeutic insight for herbal medicines. Brief Bioinform. 2014;15(5):710-733. doi:10.1093/bib/bbt035

16. Rebhan M, Chalifa-Caspi V, Prilusky J, et al. GeneCards: a novel functional genomics compendium with automated data mining and query reformulation support. Bioinformatics. 1998;14(8):656-664. doi:10.1093/bioinformatics/14.8.656

17. Amberger JS, Hamosh A. Searching Online Mendelian Inheritance in Man (OMIM): a knowledgebase of human genes and genetic phenotypes. Curr Protoc Bioinformatics. 2017;58:212. doi:10.1002/cpbi.27

18. Szklarczyk D, Gable AL, Lyon D, et al. STRING v11: protein-protein association networks with increased coverage, supporting functional discovery in genome-wide experimental datasets. Nucleic Acids Res. 2019;47(D1):D607-D613. doi:10.1093/nar/gky1131

19. Jiao X, Sherman BT, Da Huang W, et al. DAVID-WS: a stateful web service to facilitate gene/protein list analysis. Bioinformatics. 2012;28(13):1805-1806. doi:10.1093/bioinformatics/bts251

20. Grosdidier A, Zoete V, Michielin O. SwissDock, a protein-small molecule docking web service based on EADock DSS. Nucleic Acids Res. 2011;39(WebServer issue):W270-277. doi:10.1093/nar/gkr366

21. Tanji N, Markowitz GS, Fu C, et al. Expression of advanced glycation end products and their cellular receptor RAGE in diabetic nephropathy and nondiabetic renal disease. $J$ Am Soc Nephrol. 2000;11(9):1656-1666. doi:10.1681/ASN.V1191656

22. Tan RZ, Wang C, Deng C, et al. Quercetin protects against cisplatininduced acute kidney injury by inhibiting Mincle/Syk/NF-kappaB signaling maintained macrophage inflammation. Phytother Res. 2020;34(1):139-152. doi:10.1002/ptr.6507

23. Shu B, Feng Y, Gui Y, et al. Blockade of CD38 diminishes lipopolysaccharide-induced macrophage classical activation and acute kidney injury involving NF-kappaB signaling suppression. Cell Signal. 2018;42:249-258. doi:10.1016/j.cellsig.2017.10.014
24. Lei D, Chengcheng L, Xuan Q, et al. Quercetin inhibited mesangial cell proliferation of early diabetic nephropathy through the Hippo pathway. Pharmacol Res. 2019;146:104320. doi:10.1016/j.phrs.20 19.104320

25. Chen P, Shi Q, Xu X, et al. Quercetin suppresses NF-kappaB and MCP-1 expression in a high glucose-induced human mesangial cell proliferation model. Int J Mol Med. 2012;30(1):119-125. doi:10. 3892/ijmm.2012.955

26. Andres-Hernando A, Li N, Cicerchi C, et al. Protective role of fructokinase blockade in the pathogenesis of acute kidney injury in mice. Nat Commun. 2017;8:14181. doi:10.1038/ncomms14181

27. Oyagbemi AA, Akinrinde AS, Adebiyi OE, et al. Luteolin supplementation ameliorates cobalt-induced oxidative stress and inflammation by suppressing NF-KB/Kim-1 signaling in the heart and kidney of rats. Environ Toxicol Pharmacol. 2020;80:103488. doi:10.1016/j. etap. 2020.103488

28. Hong X, Zhao X, Wang G, et al. Luteolin treatment protects against renal ischemia-reperfusion injury in rats. Mediators Inflamm. 2017;2017:9783893. doi:10.1155/2017/9783893

29. Xin SB, Yan H, Ma J, et al. Protective effects of luteolin on lipopolysaccharide-induced acute renal injury in mice. Med Sci Monit. 2016;22:5173-5180. doi:10.12659/msm.898177

30. Domitrovic R, Cvijanovic O, Pugel EP, et al. Luteolin ameliorates cisplatin-induced nephrotoxicity in mice through inhibition of platinum accumulation, inflammation and apoptosis in the kidney. Toxicology. 2013;310:115-123. doi:10.1016/j.tox.2013.05.015

31. Sahu BD, Mahesh Kumar J, Sistla R. Baicalein, a bioflavonoid, prevents cisplatin-induced acute kidney injury by up-regulating antioxidant defenses and down-regulating the MAPKs and NF-kappaB pathways. PLoS One. 2015;10(7):e0134139. doi:10.1371/journal.pone.0134139

32. Li D, Shi G, Wang J, et al. Baicalein ameliorates pristane-induced lupus nephritis via activating Nrf2/HO-1 in myeloid-derived suppressor cells. Arthritis Res Ther. 2019;21(1):105. doi:10.1186/s13075019-1876-0

33. Lee EK, Kim JM, Choi J, et al. Modulation of NF-kappaB and FOXOs by baicalein attenuates the radiation-induced inflammatory process in mouse kidney. Free Radic Res. 2011;45(5):507-517. doi:10.3109/10715762.2011.555479

34. Wu K, Li H, Tian J, et al. Protective effect of baicalein on renal ischemia/reperfusion injury in the rat. Ren Fail. 2015;37(2):285-291. doi:10.3109/0886022X.2014.991999

35. Wang W, Zhou PH, Xu CG, et al. Baicalein ameliorates renal interstitial fibrosis by inducing myofibroblast apoptosis in vivo and in vitro. BJU Int. 2016;118(1):145-152. doi:10.1111/bju.13219

36. Fouqueray B, Boutard V, Philippe C, et al. Mesangial cell-derived interleukin-10 modulates mesangial cell response to lipopolysaccharide. Am J Pathol. 1995;147(1):176-182.

37. Tang TT, Wang B, Wu M, et al. Extracellular vesicle-encapsulated IL-10 as novel nanotherapeutics against ischemic AKI. Sci $A d v$. 2020;6(33):eaaz0748. doi:10.1126/sciadv.aaz0748

38. Armata HL, Golebiowski D, Jung DY, et al. Requirement of the ATM/p53 tumor suppressor pathway for glucose homeostasis. Mol Cell Biol. 2010;30(24):5787-5794. doi:10.1128/MCB.00347-10

39. Chao C, Herr D, Chun J, et al. Ser18 and 23 phosphorylation is required for p53-dependent apoptosis and tumor suppression. $E M B O$ J. 2006;25(11):2615-2622. doi:10.1038/sj.emboj.7601167

40. Wellenstein MD, Coffelt SB, Duits DEM, et al. Loss of p53 triggers WNT-dependent systemic inflammation to drive breast cancer metastasis. Nature. 2019;572(7770):538-542. doi:10.1038/s41586-019-1450-6

41. Pribluda A, Elyada E, Wiener Z, et al. A senescence-inflammatory switch from cancer-inhibitory to cancer-promoting mechanism. Cancer Cell. 2013;24(2):242-256. doi:10.1016/j.ccr.2013.06.005

42. Tang X, Wang H, Fan L, et al. Luteolin inhibits Nrf2 leading to negative regulation of the Nrf2/ARE pathway and sensitization of human lung carcinoma A549 cells to therapeutic drugs. Free Radic Biol Med. 2011;50(11):1599-1609. doi:10.1016/j.freeradbiomed.2011.03.008 
43. Li L, Luo W, Qian Y, et al. Luteolin protects against diabetic cardiomyopathy by inhibiting NF-kappaB-mediated inflammation and activating the Nrf2-mediated antioxidant responses. Phytomedicine. 2019;59:152774. doi:10.1016/j.phymed.2018.11.034

44. Kang KA, Piao MJ, Hyun YJ, et al. Luteolin promotes apoptotic cell death via upregulation of Nrf2 expression by DNA demethylase and the interaction of Nrf2 with p53 in human colon cancer cells. Exp Mol Med. 2019;51(4):1-14. doi:10.1038/s12276-019-0238-y

45. Gao JR, Jiang NN, Jiang H, et al. Effects of Qi Teng Xiao Zhuo granules on circRNA expression profiles in rats with chronic glomerulonephritis. Drug Des Devel Ther. 2019;13:1901-1913. doi:10.2147/ DDDT.S191386

46. Navarro-Nunez L, Lozano ML, Martinez C, et al. Effect of quercetin on platelet spreading on collagen and fibrinogen and on multiple platelet kinases. Fitoterapia. 2010;81(2):75-80. doi:10.1016/j.fitote.2009.08.006

47. Liu T, Yang Q, Zhang X, et al. Quercetin alleviates kidney fibrosis by reducing renal tubular epithelial cell senescence through the SIRT1/ PINK1/mitophagy axis. Life Sci. 2020;257:118116. doi:10.1016/j. lfs. 2020.118116

48. Dos Santos M, Poletti PT, Favero G, et al. Protective effects of quercetin treatment in a pristane-induced mouse model of lupus nephritis. Autoimmunity. 2018;51(2):69-80. doi:10.1080/08916934. 2018.1442828

49. Wei B, Zhang Y, Tang L, et al. Protective effects of quercetin against inflammation and oxidative stress in a rabbit model of knee osteoarthritis. Drug Dev Res. 2019;80(3):360-367. doi:10.1002/ddr.21510

50. Kim BH, Choi JS, Yi EH, et al. Relative antioxidant activities of quercetin and its structurally related substances and their effects on NF-kappaB/CRE/AP-1 signaling in murine macrophages. Mol Cells. 2013;35(5):410-420. doi:10.1007/s10059-013-0031-z
51. Yu MC, Chen JH, Lai CY, et al. Luteolin, a non-selective competitive inhibitor of phosphodiesterases 1-5, displaced [3H]-rolipram from high-affinity rolipram binding sites and reversed xylazine/ketamineinduced anesthesia. Eur J Pharmacol. 2010;627(1-3):269-275. doi:10.1016/j.ejphar.2009.10.031

52. Xagorari A, Papapetropoulos A, Mauromatis A, et al. Luteolin inhibits an endotoxin-stimulated phosphorylation cascade and proinflammatory cytokine production in macrophages. $J$ Pharmacol Exp Ther. 2001;296(1):181-187.

53. Abu-Elsaad N, El-Karef A. Protection against nonalcoholic steatohepatitis through targeting IL-18 and IL-1alpha by luteolin. Pharmacol Rep. 2019;71(4):688-694. doi:10.1016/j.pharep.2019.03.009

54. Si D, Wang Y, Zhou YH, et al. Mechanism of CYP2C9 inhibition by flavones and flavonols. Drug Metab Dispos. 2009;37(3):629-634. doi:10.1124/dmd.108.023416

55. Shieh DE, Liu LT, Lin CC. Antioxidant and free radical scavenging effects of baicalein, baicalin and wogonin. Anticancer Res. 2000;20 (5A):2861-2865.

56. Tarrago T, Kichik N, Claasen B, et al. Baicalin, a prodrug able to reach the CNS, is a prolyl oligopeptidase inhibitor. Bioorg Med Chem. 2008;16(15):7516-7524. doi:10.1016/j.bmc.2008.04.067

57. Nakahama K, Morita I, Murota S. Effects of endogenously produced arachidonic acid metabolites on rat mesangial cell proliferation. Prostaglandins Leukot Essent Fatty Acids. 1994;51(3):177-182. doi:10.1016/0952-3278(94)90131-7
Journal of Inflammation Research

\section{Publish your work in this journal}

The Journal of Inflammation Research is an international, peerreviewed open-access journal that welcomes laboratory and clinical findings on the molecular basis, cell biology and pharmacology of inflammation including original research, reviews, symposium reports, hypothesis formation and commentaries on: acute/chronic inflammation; mediators of inflammation; cellular processes; molecular

\section{Dovepress}

mechanisms; pharmacology and novel anti-inflammatory drugs; clinical conditions involving inflammation. The manuscript management system is completely online and includes a very quick and fair peerreview system. Visit http://www.dovepress.com/testimonials.php to read real quotes from published authors. 